\title{
Energy partition patterns and plasma metabolomics profiles of modern growing pigs under different ambient temperatures: modelling and integrative analysis
}

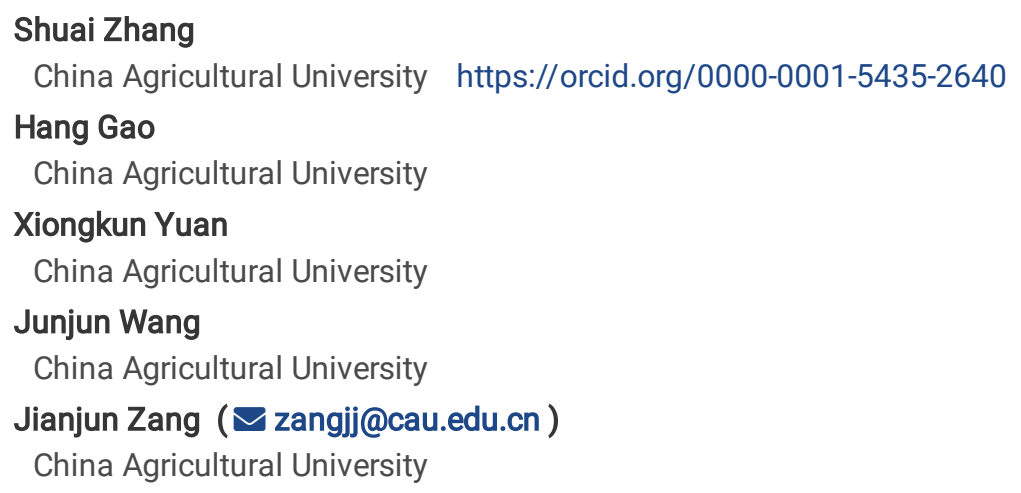

Keywords: ambient temperature, energy partition pattern, plasma metabolomics profile, modern growing pigs

Posted Date: July 27th, 2020

DOI: https://doi.org/10.21203/rs.3.rs-47836/v1

License: (1) This work is licensed under a Creative Commons Attribution 4.0 International License. Read Full License 


\section{Abstract \\ Background}

Most of the studies focusing on energy partition patterns of growing pigs and the related mechanisms under different ambient temperatures were carried out during 1970s to early 2000s. With the rapid developments in pig breeding, research updates on such topics concerning modern growing pigs have been absent in the last decade. This study developed mathematical models to predict the energy partition patterns of modern growing pigs with different BWs at gradient-ambient temperatures, and investigated the underlying changes in plasma under such conditions based on the integrative analysis of indirect calorimetry and non-target metabolomics profiling.

\section{Methods}

In trial 1, 36 barrows with initial BW of $26.4 \pm 1.9 \mathrm{~kg}$ were allotted to a $6 \times 6$ Latin Square Design with ambient temperatures set as $18{ }^{\circ} \mathrm{C}$, $21^{\circ} \mathrm{C}, 23^{\circ} \mathrm{C}, 27^{\circ} \mathrm{C}, 30^{\circ} \mathrm{C}$, and $32^{\circ} \mathrm{C}$. In trial 2,24 barrows with initial BW of $64.2 \pm 3.1 \mathrm{~kg}$ were allotted to a $4 \times 6$ Youden Square Design with ambient temperatures set as $18{ }^{\circ} \mathrm{C}, 23^{\circ} \mathrm{C}, 27^{\circ} \mathrm{C}$, and $32^{\circ} \mathrm{C}$. Each pig was kept in individual metabolic crate and consumed feed ad libitum, then transferred into the respiration chamber for 5-day indirect calorimetry assay and 1-day fasting. The energy and nitrogen balance as well as energy utilization efficiency were determined, and mathematical models were developed to predict the voluntary feed intake $(\mathrm{VFI})$, metabolizable energy intake $\left(\mathrm{ME}_{\mathrm{i}}\right)$ and energy retention as protein $\left(R E_{\mathrm{P}}\right)$ and lipid $\left(R E_{\mathrm{L}}\right)$ using $B W$ and ambient temperature $(\mathrm{T})$ as predictors. Moreover, plasma samples were collected from pigs at $25 \mathrm{~kg}$ under different temperatures, and the levels of hormone and metabolites in plasma were determined using biochemical and the metabolomics approaches, respectively.

\section{Results}

As the ambient temperature increased from $18{ }^{\circ} \mathrm{C}$ to $32{ }^{\circ} \mathrm{C}$, the $\mathrm{VFI}, \mathrm{ME}_{\mathrm{i}}, \mathrm{N}$ intake and retention, total heat production (THP) and $\mathrm{RE} \mathrm{P}_{\mathrm{p}}$ of growing pigs at $25 \mathrm{~kg}$ all linearly decreased $(P<0.05)$. There were interaction effects between $\mathrm{BW}$ and $\mathrm{T}$ on $\mathrm{VFI}, \mathrm{ME}_{\mathrm{i}}, \mathrm{N}$ balance, adjusted THP, $\mathrm{RE}_{\mathrm{P}}$ and $\mathrm{RE}_{\mathrm{L}}(P<0.05)$. Parallel curves were observed between the VFI model developed in the current study and that built previously, but our $\mathrm{ME}_{\mathrm{i}}$ model showed discrepancy from the previous model especially at high temperatures. The models simulated for $\mathrm{RE}_{\mathrm{P}}$ and $\mathrm{RE} \mathrm{E}_{\mathrm{L}}$ are: $\mathrm{RE}_{\mathrm{p}}\left(\mathrm{kJ} / \mathrm{kg} \mathrm{BW}^{0.6} / \mathrm{d}\right)=-233.38-67.28 \times \mathrm{BW}^{0.6}+3.16 \times\left(\mathrm{BW}^{0.6}\right)^{2}+39.58 \times \mathrm{T}-0.76 \times \mathrm{T}^{2}-0.26 \times \mathrm{BW}^{0.6} \times \mathrm{T}+0.26 \times \mathrm{ME}_{\mathrm{i}}$, and $\mathrm{RE}_{\mathrm{L}}$ $\left(\mathrm{kJ} / \mathrm{kg} \mathrm{BW}^{0.6} / \mathrm{d}\right)=-465.10+8.10 \times \mathrm{BW}^{0.6}-1.03 \times\left(B W^{0.6}\right)^{2}-53.73 \times \mathrm{T}+1.34 \times \mathrm{T}^{2}+0.67 \times \mathrm{BW}^{0.6} \times \mathrm{T}+0.69 \times \mathrm{ME}_{\mathrm{i}}$, respectively. The cortisol and thyroid hormone levels in plasma linearly decreased as the ambient temperature increased from $18{ }^{\circ} \mathrm{C}$ to $32^{\circ} \mathrm{C}$, and 13 compounds were identified through metabolomics analysis, including up-regulated metabolites involved in fatty acid metabolism such as adrenic acid and down-regulated metabolites involved in amino acid metabolism such as spermidine at $32{ }^{\circ} \mathrm{C}$.

\section{Conclusion}

These results suggested that pigs at heavier body weight were more sensitive to high temperatures on energy intake and partition, and pigs with modern genotypes could be more sensitive to heat stress than pigs with older genotypes. Most of the identified metabolites altered under high ambient temperatures are associated with suppressed fatty acid oxidation and elevated lipogenesis and protein degradation.

\section{Background}

Moderate ambient temperature in house is the prerequisite for animals to maintain health and performance. Especially in the modern large-scaled and high-efficient livestock production system, high or low temperature is one of the major stressors that animals face. Under thermoneutral zone, the dietary energy intake is used by animals to meet the requirements of maintenance, physiological activity, and production (e.g. growth, reproduction) [1]. When the ambient temperature is below the lower critical temperature (LCT) of the thermoneutral zone, heat production (HP) increases in warm-blooded animals such as pigs to meet the additional energy requirement of

thermoregulation, with more feed are consumed to maintain the growth performance [2]. On the other hand, the feed intake, HP, and energy retention (RE) of pigs will decrease when the ambient temperature is above the upper critical temperature (UCT) of the thermoneutral zone, along with the reduced locomotion [3]. Those threshold temperatures vary depending on animal-related factors such as genotype, body weight (BW), and physiological stage [4].

Page $2 / 22$ 
The research on effects of ambient temperature on patterns of physiological changes in livestock started on 1940s, with the application of artificial climate warehouse to mimic the environment in animal houses. In pigs, many studies were conducted from 1970 s to early 2000 s focusing on energy partition patterns under different temperatures using the climate-controlled open-circuit respiratory chamber [1-3, 59]. With the development of animal breeding, modern pigs have great progress in productivity (e.g. quick weight gain and higher reproductivity), but their bodies become more sensitive to adverse environment such as changes in ambient temperatures [10]. Therefore, researches were carried out in recent years concerning about the alternations and alleviation strategies of modern pigs under heat or cold stress. However, there is few updates on patterns of energy balances in modern pigs at different ambient temperatures especially in the last decade.

Indirect calorimetry is the traditional non-invasion approach to determine the HP of the subjects, and has been widely used in livestock to monitor and model the energy partition patterns together with the analysis of feed intake and urine and feces outputs [11]. Moreover, the metabolic changes underlying the energy reactions has received more attention in recent years, which could directly reflect the regulation and adaptation of the animals to the surrounding conditions, and may provide novel diagnostic tools or biomarkers with high sensitivity towards the adverse effects [4]. The non-targeting metabolomics is an ideal approach to analyze the comprehensive changes in metabolites of the subjects, and has never been used to explore the relationships between metabolites alternation and the patterns of energy expenditure or substrate oxidation of growing pigs under various ambient temperatures.

Therefore, the objectives of this study were to determine and establish models for the energy partition patterns of modern growing pigs with different BWs at gradient-ambient temperatures, and to identify the underlying changes in energy metabolism under such conditions based on the integrative analysis of indirect calorimetry and non-target plasma metabolomics profiling.

\section{Methods}

The Institutional Animal Care and Use Committee of China Agricultural University (Beijing, China) approved all protocols used in this experiment.

\section{Equipment for indirect calorimetry}

Six open-circuit respiration chambers $\left(1.4 \times 2.7 \times 2.1 \mathrm{~m}^{3}\right)$ located in the FengNing Swine Research Unit of China Agricultural University (Academician Workstation in Chengdejiuyun Agricultural \& Livestock Co., Ltd) were used to conduct the indirect calorimetry, which were described in details in previous studies $[12,13]$ with the exception of temperature settings. The relative humidity was controlled at 70$75 \%$, and the air velocity was controlled at $0.1 \mathrm{~m} / \mathrm{s}$. Oxygen content was measured with a paramagnetic differential analyzer (Oxymat $6 \mathrm{E}$,

Siemens, Munich, Germany), whereas $\mathrm{CO}_{2}$ and $\mathrm{CH}_{4}$ contents were measured with infrared analyzers (Ultramat $6 \mathrm{E}$, Siemens). The analyzers had a range of $19.5-21.0 \%$ for $\mathrm{O}_{2}, 0-1 \%$ for $\mathrm{CO}_{2}$, and $0-0.1 \%$ for $\mathrm{CH}_{4}$, with a sensitivity of $0.2 \%$. Gas concentrations in each chamber were measured at 5-min intervals. The ethanol combustion experiment was used to check the accuracy of the chamber in measuring gaseous exchange.

\section{Animals, diets and experiment design}

Two animal trials were conducted in this study. In Trial 1, 36 healthy growing barrows (Duroc $\times$ Large White $\times$ Landrace) with initial BW of $26.4 \pm 1.9 \mathrm{~kg}$ were allotted to a $6 \times 6$ Latin Square Design in 6 consecutive periods. In each period, 6 pigs were moved into 6 open-circuit respiration chambers with ambient temperatures setting as $18{ }^{\circ} \mathrm{C}, 21^{\circ} \mathrm{C}, 23^{\circ} \mathrm{C}, 27^{\circ} \mathrm{C}, 30^{\circ} \mathrm{C}$, and $32^{\circ} \mathrm{C}$, respectively. In Trial 2,24 healthy growing barrows (Duroc $\times$ Large White $\times$ Landrace) with initial BW of $64.2 \pm 3.1 \mathrm{~kg}$ were allotted to a $4 \times 6$ Youden Square Design in 6 consecutive periods. In each period, 4 pigs were moved into 4 open-circuit respiration chambers (only 4 chambers were available during Trial 2) with ambient temperatures setting as $18{ }^{\circ} \mathrm{C}, 23^{\circ} \mathrm{C}, 27^{\circ} \mathrm{C}$, and $32^{\circ} \mathrm{C}$, respectively.

Two diets based on corn-soybean meal were formulated to meet or exceed the nutrient requirements for growing pigs at $25 \mathrm{~kg}$ and $65 \mathrm{~kg}$ according to the recommendations by NRC [14], and were applied in Trial 1 and 2, respectively. The analyzed chemical compositions of those two diets were shown in Table 1. 
Table 1

Ingredient compositions and analyzed chemical components of the complete diets fed to barrows at different growth stages used in Exp.1 and Exp.2 (as-fed basis)

\begin{tabular}{|c|c|c|}
\hline \multirow[t]{2}{*}{ Items } & \multicolumn{2}{|c|}{ Growth stage } \\
\hline & $25 \mathrm{~kg}$ & $65 \mathrm{~kg}$ \\
\hline \multicolumn{3}{|l|}{ Ingredients, \% } \\
\hline Corn & 77.13 & 80.39 \\
\hline Soybean meal & 20.00 & 17.00 \\
\hline Dicalcium phosphate & 0.90 & 0.80 \\
\hline Limestone & 0.75 & 0.65 \\
\hline Sodium chloride & 0.35 & 0.35 \\
\hline Premix $^{1}$ & 0.50 & 0.50 \\
\hline Lysine-HCl & 0.21 & 0.17 \\
\hline DL-Methionine & 0.11 & 0.09 \\
\hline L-Threonine & 0.02 & 0.02 \\
\hline L-Tryptophan & 0.03 & 0.03 \\
\hline \multicolumn{3}{|c|}{ Analyzed nutrient levels, $\%$} \\
\hline Dry matter & 89.50 & 89.60 \\
\hline Gross energy, MJ/kg & 16.38 & 16.67 \\
\hline Crude protein & 17.78 & 16.20 \\
\hline Ether extract & 2.89 & 2.57 \\
\hline Ash & 4.72 & 3.34 \\
\hline \multicolumn{3}{|c|}{ 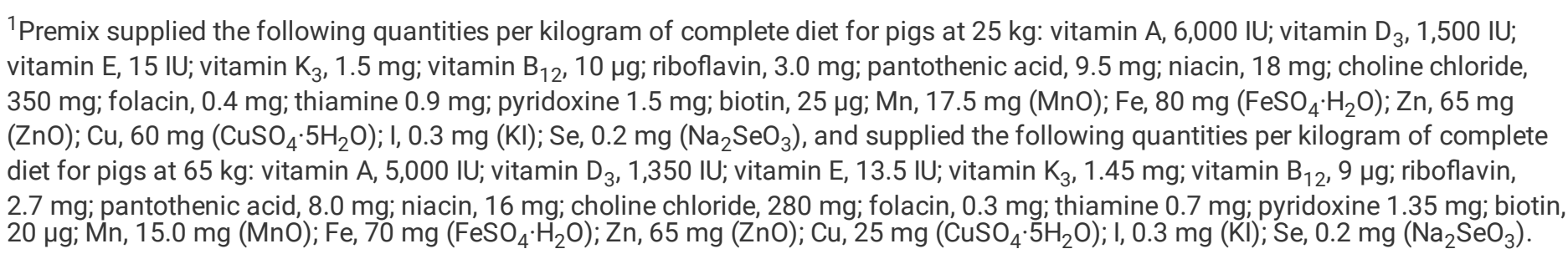 } \\
\hline
\end{tabular}

The specific experiment procedures were kept the same in both animal trials. In each period, pigs were kept individually in stainless-steel metabolism crates $\left(1.2 \times 0.5 \times 0.6 \mathrm{~m}^{3}\right)$ equipped with feeders and low-pressure nipple drinkers, and had a 7-d adaption period for crates and diets in the thermoneutrual-controlled room $\left(24 \pm 1^{\circ} \mathrm{C}\right)$. On day 8 , pigs were weighted and moved into the chambers for 5 days to measure the gases exchanges and calculate the total heat production (THP). Based on the feed intake during adaption, feed was supplied twice daily (08:30 and 15:30) ad libitum to pigs in chambers, and pigs had free access to water. For each pig, the total feces, urine output and wasted feed were collected twice daily from 08:00 to 08:30, and from 15:00 to 15:30, when the production of $\mathrm{CO}_{2}, \mathrm{CH}_{4}$ and $\mathrm{O}_{2}$ consumption were expelled in the calculation of daily HP, to determine the energy intakes of each day. On day 13 , pigs were weighted in the morning and deprived of feed and fasted for $24 \mathrm{~h}$, and the total urine output were collected during the fasting period to determine the fasting heat production (FHP), as an estimation of the energy used for maintenance [12]. On the morning of day 14, pigs were moved out the chambers and weighted again.

\section{Sample collection}

The daily urine output of each pig was collected into buckets with $50 \mathrm{~mL} 6 \mathrm{~N} \mathrm{HCl}$ and sieved thereafter through multilayer gauze into plastic bottles and stored in $-20^{\circ} \mathrm{C}$. The 5 -day total urine collection and urine collection during the fasting period were quantified for each pig respectively, and a subsample of $50 \mathrm{~mL}$ for each pig was saved from the thoroughly mixed 5-day total collection for further analysis. The daily feces output of each pig was collected into plastic bags and immediately stored at $-20^{\circ} \mathrm{C}$, then the 5 -day total feces collection 
was thawed, mixed, and weighted, and a subsample of $350 \mathrm{~g}$ for each pig was oven-dried for $72 \mathrm{~h}$ at $65^{\circ} \mathrm{C}$ to calculate the moisture content. Feed samples and subsamples of dried feces were finely ground through a $1 \mathrm{~mm}$ sieve prior to chemical analysis.

Blood samples of pigs in Trial 1 were collected from precaval vein into the heparinized tubes on day 13 morning during fasting, and then were centrifuged (Biofuge22R; Heraeus, Hanau, Germany) at 3,000 $\times g$ for $10 \mathrm{~min}$ at $4{ }^{\circ} \mathrm{C}$. The supernatants were transferred to storage tubes, frozen in liquid nitrogen, and stored at $-80^{\circ} \mathrm{C}$ for further assays.

\section{Chemical analysis and calculation}

All chemical analysis of samples was conducted in duplicates and repeated if the duplicates differed by more than $5 \%$. The gross energy (GE) in feed, feces, and urine samples was determined using an isoperibol calorimeter (Parr 6400 Calorimeter, Moline, IL, USA) with a standard reference of benzoic acid. For feed samples, the contents of dry matter (DM), crude protein (CP), ether extract (EE), and ash were determined following the methods of AOAC [15]. For fecal samples, the contents of DM and CP were determined. For urine samples, the nitrogen $(\mathrm{N})$ content was measured according to Li et al. [13].

The dry matter intake (DMi) of each pig from day 8 to day 12 was calculated as the product of feed intake and the DM content of the diets. The GE intake was calculated as the product of actual DMi during the 5-day collection period and the GE values of the diets. The digestible energy $(\mathrm{DE})$ intake and metabolizable energy intake $\left(\mathrm{ME}_{\mathrm{i}}\right)$ was calculated as the difference between $\mathrm{GE}$ intake and the energy loss in feces, and the difference between DE intake and the energy loss in urine and methane, respectively. Methane energy was calculated using the methane volume and a conversion factor of $39.4 \mathrm{~kJ} / \mathrm{L}[16]$.

The average daily THP was calculated according to the following equation based on the gas exchanges $\left(\mathrm{O}_{2}\right.$ consumption and productions of $\mathrm{CO}_{2}$ and $\mathrm{CH}_{4}$ ) during day 8 to day 12 recorded at 5-min intervals and then averaged and extrapolated to a 24-h period:

$\mathrm{HP}(\mathrm{kJ})=16.18 \times \mathrm{O}_{2}(\mathrm{~L})+5.02 \times \mathrm{CO}_{2}(\mathrm{~L})-2.17 \times \mathrm{CH}_{4}(\mathrm{~L})-5.99 \times$ urinary $\mathrm{N}(\mathrm{g})[16]$.

The FHP was calculated using the same equation, but the 24-h FHP was predicted from the 8-h HP after feed deprivation from 22:00 to 06:00 during the last day of each period, which was then extrapolated to the 24-h period [13]. The RE was calculated as the difference between $M E_{i}$ and THP, while the $R E$ as protein $\left(R E_{P}\right)$ was calculated as $N$ retention $(g / d) \times 6.25 \times 23.86(\mathrm{~kJ} / \mathrm{g})$, and the $R E$ as lipid $\left(P E_{L}\right)$ was calculated as the difference between RE and $R E_{p}$. The net energy (NE) intake was calculated as the sum of RE and maintenance energy estimated by FHP [17]. Moreover, the oxidation of carbohydrates $(\mathrm{OXCHO})$ and fat (OXF) were calculated by the method described by Chwalibog et al. according to the following equations [18]:

$\mathrm{OXCHO}(\mathrm{kJ} / \mathrm{d})=\left[-2.968 \times \mathrm{O}_{2}(\mathrm{~L})+4.147 \times \mathrm{CO}_{2}(\mathrm{~L})-1.761 \times \mathrm{CH}_{4}(\mathrm{~L})-2.446 \times\right.$ Urinary $\left.\mathrm{N}(\mathrm{g})\right] \times 17.58$

$\operatorname{OXF}(\mathrm{kJ} / \mathrm{d})=\left[1.719 \times \mathrm{O}_{2}(\mathrm{~L})-1.719 \times \mathrm{CO}_{2}(\mathrm{~L})-1.719 \times \mathrm{CH}_{4}(\mathrm{~L})-1.963 \times\right.$ Urinary $\left.\mathrm{N}(\mathrm{g})\right] \times 39.76$

The rates of DE:GE, ME:DE and NE:ME were then calculated based on the calculations of GE, DE, ME, and NE intakes. The respiratory quotient (RQ) was calculated as the ration between $\mathrm{CO}_{2}$ production and $\mathrm{O}_{2}$ consumption. All the energy balance indexes were present on the metabolic body weight (per kg BW ${ }^{0.60}$ ) basis.

\section{Hormone and biochemical marker assays in serum}

The concentrations of cortisol, insulin, triiodothyronine $\left(T_{3}\right)$ and thyroxine $\left(T_{4}\right)$ in serum were analyzed using radioimmunoassay, while the activities of growth hormone $(\mathrm{GH})$ and glucagon in serum were analyzed using commercial enzyme-linked immunosorbent assay (ELISA) kits (Nanjing Jiancheng Bioengineering Institute, Nanjing, China) following the manufacturer's guides. The levels of albumin, glutamicpyruvic transaminase (ALT), glutamic oxalacetic transaminase (AST), globulin, high density lipoprotein (HDL), low density lipoprotein (LDL), total cholesterol (TC), triglyceride (TG), total protein and urea in serum were determined using the automatic biochemical analyzer (7170, Hitachi Corp., Tokyo, Japan) with corresponding commercial kits (Nanjing Jiancheng Bioengineering Institute) following the manufacturer's guides.

\section{Non-target metabolomics profiling in plasma and data analysis}

Plasma samples from pigs kept under the ambient temperatures of $18{ }^{\circ} \mathrm{C}, 23^{\circ} \mathrm{C}$ and $32{ }^{\circ} \mathrm{C}$ (defined as low, neutral and high ambient temperature group, respectively) were used for non-target metabolomics profiling assay, followed the procedures described by Liu et al. with some modifications [19]. 
Briefly, $100 \mu \mathrm{L}$ plasma samples were added into $400 \mu \mathrm{L}$ ice-cold extraction mix (methanol: acetonitrile $=1: 1$, v: v). After vortexing for $10 \mathrm{~s}$, the mixture was centrifuged (Eppendorf, Germany) at $15,000 \times g$ for 10 min at $4{ }^{\circ} \mathrm{C}$ to remove protein, and $400 \mu \mathrm{L}$ supernatant was collected and evaporated to dryness using a vacuum concentrator (Concentrator PLUS, Eppendorf). The resulting dry residues were resuspended in $200 \mu \mathrm{L}$ recovery solution (water: methanol $=4: 1$ ), vortexed and centrifuged again at $15,000 \times g$ for $10 \mathrm{~min}$ at $4{ }^{\circ} \mathrm{C}$. The supernatant was filtered through a $0.22 \mu \mathrm{m}$ membrane and transferred to sampler vials to be analyzed on a UPLC-MS system (UPLC, ACQUITY UPLC H-Class PLUS Bio System, Waters Corporation, MA, USA; MS, Q-Exactive, Termo Scientific, NJ, USA) equipped with a heated electrospray ionization (HESI) source. The UPLC separation was operated on a BEH C18 column $(2.1 \times 100 \mathrm{~mm}, 1.7 \mu \mathrm{m}, \mathrm{Waters}$ Corporation), with mobile phase comprised of $0.1 \%$ formic acid water solution $(A)$ and $0.1 \%$ formic acid acetonitrile solution (B), flow rate set as $0.3 \mathrm{~mL} / \mathrm{min}$, column temperature set at $35^{\circ} \mathrm{C}$, and injection volume set as $5 \mu \mathrm{L}$. The MS analysis was performed in an electrospray ionization positive mode, and data were acquired with full scan using a mass resolution of 70,000 and a scan range of 50 to $750 \mathrm{~m} / \mathrm{z}$. For MS/MS analysis, an isolation window of $2.0 \mathrm{~m} / \mathrm{z}$ and a mass resolution of 17,500 were selected.

\section{Statistical Analysis}

For the metabolomics data, the software SIEVE 2.1 (Thermo Scientific) and Progenesis QI (Nonlinear Dynamics, Waters Corporation) were used for raw data processing, including background subtraction, peak alignment, identification, and normalization with Pareto scaling to get the standardized relative abundance of the metabolites in different samples. Then principal components analysis (PCA) was conducted using SIMCA-P 13 software (Umetrics, Umea, Sweden), and one-way ANOVA was conducted using JMP Pro 14.3.0 (SAS Institute Inc., Carry, NC, USA) to compare the abundance of metabolites among different treatments. The metabolites with fold change > $1.5, \mathrm{CV}<20 \%$ and $P<0.05$ were selected for further identification by comparison of the ion features in the experimental samples to the chemical standard entries in reference libraries (METLIN, HMDB and KEGG) that included retention time, molecular weight (m/z) as well as their associated MS/MS spectra. Boxplot was plotted using the ggplot2 package in R software (http://cran.r-project.org/, version 4.0.2) to illustrate the concentrations of the compounds that identified with significantly difference among the three treatments. Those compounds were then imported into the module of pathway analysis in Metaboanalyst 3.0

(https://www.metaboanalyst.ca/MetaboAnalyst/upload/PathUploadView.xhtml) to generate the pathway topology analysis. The metabolic pathway with impact value greater than 0.1 was characterized as the significantly relevant pathway [19].

For the other data, normality and homogeneity of variance were checked using the normal probability plot and residual plot from JMP Pro 14.3.0, and outliers were removed before further analysis. For $\mathrm{N}$ and energy balance data and serumal hormone and biochemical markers data collected in Trial 1, a statistical model including ambient temperature as the only fixed effect and chamber as the random effect was fitted using the Fit Model function in JMP Pro 14.3.0. Tukey's HSD test was used to separate the least square means among treatments with significantly different effects. Moreover, linear and quadratic effects of increased ambient temperature on responses were tested by polynomial contrast using the Fit $Y$ by $X$ function in JMP Pro 14.3.0. To better illustrate the comprehensive effects of ambient temperature and pig BW, the $\mathrm{N}$ and energy balance data collected in Trial 2 were combined with some corresponding data (those under the same temperature settings) in Trial 1 for analysis. A statistical model included the main effects of ambient temperature $\left(18^{\circ} \mathrm{C}, 23^{\circ} \mathrm{C}, 27^{\circ} \mathrm{C}\right.$, and $\left.32^{\circ} \mathrm{C}\right)$ and BW (25 kg and $\left.65 \mathrm{~kg}\right)$, and their interaction effect as fixed effects, and included the chamber as the random effect. The Fit Model function in JMP Pro 14.3.0 was used to conduct the two-way ANOVA, and Tukey's HSD test was used to separate the least square means among treatments with significantly different main effects only when the interaction effect was not significant. Moreover, equations were developed to predict the voluntary feed intake (VFI), $M E_{i}, R E_{P}$ and $R E_{L}$ using metabolic body weight (BW 0.6 ) and ambient temperature as predictors based on some modifications of the equations from Quiniou et al. [20]:

VFI or $\mathrm{ME}_{\mathrm{i}}=\mathrm{a}+\mathrm{b} \times \mathrm{BW}^{0.6}+\mathrm{c} \times\left(B \mathrm{~W}^{0.6}\right)^{2}+\mathrm{d} \times \mathrm{T}+\mathrm{e} \times \mathrm{T}^{2}+\mathrm{f} \times \mathrm{BW}^{0.6} \times \mathrm{T}$,

$R E_{P}$ or $R E_{L}=a+b \times B W^{0.6}+c \times\left(B W^{0.6}\right)^{2}+d \times T+e \times T^{2}+f \times B W^{0.6} \times T+g \times M E_{i}$

All the data obtained in Trial 1 and 2 were combined and used for model development. Covariance analysis was used to further confirm the quadratic effects of BW and T on response variables, and coefficients were estimated by Gauss-Newton analysis using the Nonlinear Modelling function in JMP Pro 14.3.0. The predicted vs. actual plot and predicted vs. residuals plot were used to check the goodness of fit of the models. For all the analysis, $P<0.05$ was considered as significantly different.

\section{Results}

\section{Energy partition in pigs at $25 \mathrm{~kg}$ under different ambient temperatures}


The energy partition of modern growing pigs at $25 \mathrm{~kg}$ kept at $18^{\circ} \mathrm{C}, 21^{\circ} \mathrm{C}, 23^{\circ} \mathrm{C}, 27^{\circ} \mathrm{C}, 29^{\circ} \mathrm{C}$ and $32{ }^{\circ} \mathrm{C}$ were present in Table 2 . As the ambient temperature increased from $18{ }^{\circ} \mathrm{C}$ to $32^{\circ} \mathrm{C}$, the $\mathrm{DMi}, \mathrm{N}$ intake, fecal $\mathrm{N}$ output, $\mathrm{N}$ retention, $\mathrm{ME}_{\mathrm{i}}, \mathrm{THP}^{\circ}$ and $\mathrm{RE} \mathrm{E}_{\mathrm{P}}$ of growing pigs at $25 \mathrm{~kg}$ all showed linearly and quadratically decreased pattern $(P<0.05)$. Specifically, pigs raised at $18^{\circ} \mathrm{C}$ had greater $\mathrm{DMi}, \mathrm{N}$ intake, $\mathrm{N}$ retention, $\mathrm{ME}_{\mathrm{i}}$, THP and $\mathrm{RE}_{\mathrm{P}}$ compared with those raised at $32^{\circ} \mathrm{C}$, while pigs kept at $18{ }^{\circ} \mathrm{C}$ also had greater THP compared with those kept at $29^{\circ} \mathrm{C}(P<0.05)$. Moreover, pigs raised at $18{ }^{\circ} \mathrm{C}$ and $21^{\circ} \mathrm{C}$ exhibited greater fecal $\mathrm{N}$ output than those raised at $27^{\circ} \mathrm{C}$, and pigs kept at $23^{\circ} \mathrm{C}$ also exhibited greater $\mathrm{RE}_{\mathrm{P}}$ than those kept at $32^{\circ} \mathrm{C}(P<0.05)$. On the other hand, as the ambient temperature increased from $18{ }^{\circ} \mathrm{C}$ to $32{ }^{\circ} \mathrm{C}$, the $\mathrm{OXCHO}$ and $\mathrm{RQ}$ at fasting state linearly and quadratically increased, with pigs under $32{ }^{\circ} \mathrm{C}$ showed greater $\mathrm{OXCHO}$ and $\mathrm{RQ}$ during fasting compared with pigs under $18{ }^{\circ} \mathrm{C}, 21^{\circ} \mathrm{C}$ and $23^{\circ} \mathrm{C}(P<0.05)$. Otherwise, no significant changes were observed on other parameters related to energy balance and utilization under different ambient temperatures $(P>0.05)$. 
Table 2

aNitrogen and energy balance of barrows at $25 \mathrm{~kg}$ kept at different ambient temperatures ${ }^{1}$.

\begin{tabular}{|c|c|c|c|c|c|c|c|c|c|c|}
\hline \multirow[t]{2}{*}{ Items } & \multicolumn{6}{|c|}{ Ambient temperature $\left({ }^{\circ} \mathrm{C}\right)$} & \multirow[t]{2}{*}{ SEM } & \multicolumn{3}{|l|}{$P_{\text {-value }}{ }^{7}$} \\
\hline & 18 & 21 & 23 & 27 & 29 & 32 & & ANOVA & Linear & Quadratic \\
\hline Average body weight, kg & 28.7 & 28.7 & 27.0 & 28.7 & 28.8 & 28.7 & 0.79 & 0.587 & 0.709 & 0.622 \\
\hline Dry matter intake, kg/d & $1.32^{\mathrm{a}}$ & $1.24^{\mathrm{ab}}$ & $1.21^{\mathrm{ab}}$ & $1.05^{\mathrm{ab}}$ & $1.16^{\mathrm{ab}}$ & $0.99^{b}$ & 0.06 & 0.030 & < 0.001 & 0.002 \\
\hline \multicolumn{11}{|l|}{ Nitrogen balance, $\mathrm{g} / \mathrm{d}$} \\
\hline Intake & $41.9^{a}$ & $39.3^{\mathrm{ab}}$ & $38.4^{\mathrm{ab}}$ & $33.4^{\mathrm{ab}}$ & $36.8^{\mathrm{ab}}$ & $31.6^{\mathrm{b}}$ & 2.05 & 0.030 & $\begin{array}{l}< \\
0.001\end{array}$ & 0.002 \\
\hline Fecal output & $6.7^{a}$ & $6.6^{a}$ & $5.9^{a b}$ & $4.3^{b}$ & $4.6^{\mathrm{ab}}$ & $4.8^{\mathrm{ab}}$ & 0.47 & 0.004 & $\begin{array}{l}< \\
0.001\end{array}$ & $<0.001$ \\
\hline Urine output & 5.4 & 4.6 & 3.2 & 4.9 & 6.0 & 3.8 & 0.92 & 0.327 & 0.867 & 0.926 \\
\hline Retention & $29.8^{a}$ & $28.0^{\mathrm{ab}}$ & $29.2^{\mathrm{ab}}$ & $24.7^{\mathrm{ab}}$ & $26.2^{\mathrm{ab}}$ & $21.7^{b}$ & 1.73 & 0.040 & < 0.001 & 0.003 \\
\hline \multicolumn{11}{|l|}{ Energy balance, $\mathrm{kJ} / \mathrm{kg} \mathrm{BW}^{0.6} / \mathrm{d}$} \\
\hline ME intake & $2699^{a}$ & $2510^{\mathrm{ab}}$ & $2555^{\mathrm{ab}}$ & $2197^{a b}$ & $2426^{a b}$ & $2035^{b}$ & 133.3 & 0.025 & $\begin{array}{l}< \\
0.001\end{array}$ & 0.004 \\
\hline Total heat production & $1133^{a}$ & $1093^{a b}$ & $1075^{\mathrm{ab}}$ & $1000^{a b}$ & $947^{b}$ & $925^{b}$ & 55.2 & 0.004 & 0.002 & 0.011 \\
\hline $\begin{array}{l}\text { Oxidation of carbohydrates fed } \\
\text { state }^{2}\end{array}$ & 1304 & 1330 & 1550 & 1236 & 1100 & 1192 & 124.2 & 0.084 & 0.168 & 0.329 \\
\hline Oxidation of fat fed state ${ }^{3}$ & -304 & -361 & -587 & -370 & -274 & -263 & 119.1 & 0.197 & 0.388 & 0.331 \\
\hline Total heat production adjusted 4 & 1010 & 1063 & 1034 & 1123 & 954 & 1069 & 86.8 & 0.629 & 0.875 & 0.953 \\
\hline Fasting heat production (FHP) & 716 & 713 & 628 & 713 & 666 & 698 & 48.6 & 0.809 & 0.758 & 0.748 \\
\hline $\begin{array}{l}\text { Oxidation of carbohydrates fast } \\
\text { state }^{2}\end{array}$ & $189^{\mathrm{b}}$ & $225^{\mathrm{b}}$ & $210^{\mathrm{b}}$ & $283^{a b}$ & $267^{\mathrm{ab}}$ & $404^{a}$ & 44.9 & 0.014 & 0.002 & 0.004 \\
\hline Oxidation of fat fast state ${ }^{3}$ & 472 & 465 & 392 & 400 & 339 & 300 & 62.0 & 0.233 & 0.017 & 0.058 \\
\hline Energy retention (RE) & 1565 & 1417 & 1480 & 1202 & 1478 & 1145 & 143.9 & 0.193 & 0.059 & 0.172 \\
\hline
\end{tabular}

a,b Within a row means with different superscript letters differ at $P<0.05$.

${ }^{1}$ ANOVA, analysis of variance; DE, digestible energy; GE, gross energy; ME: metabolizable energy; NE: net energy; SEM, standard error of the mean.

${ }^{2}$ Oxidation of carbohydrates $(\mathrm{kJ} / \mathrm{kg} \mathrm{BW} 0.6 /$ day $)=\left[-2.968 \times \mathrm{O}_{2}(\mathrm{~L})+4.147 \times \mathrm{CO}_{2}(\mathrm{~L})-1.761 \times \mathrm{CH}_{4}(\mathrm{~L})-2.446 \times \mathrm{Urinary} \mathrm{N}^{\mathrm{g}}(\mathrm{g})\right] \times$ $17.58 / \mathrm{BW}^{0.6}$.

${ }^{3}$ Oxidation of fat $(\mathrm{kJ} / \mathrm{kg} \mathrm{BW} 0.6 /$ day $)=\left[1.719 \times \mathrm{O}_{2}(\mathrm{~L})-1.719 \times \mathrm{CO}_{2}(\mathrm{~L})-1.719 \times \mathrm{CH}_{4}(\mathrm{~L})-1.963 \times \mathrm{Urinary} \mathrm{N}(\mathrm{g})\right] \times 39.76 / \mathrm{BW}^{0.6}$.

${ }^{4}$ Total heat production adjusted = Total heat production was adjusted to the same ME intake at $2.4 \mathrm{MJ} \mathrm{ME} / \mathrm{kg} \mathrm{BW} 0.6 / \mathrm{d}$.

${ }^{5} \mathrm{RE}_{\mathrm{P}}=$ Energy retention as protein $(\mathrm{kJ} / \mathrm{kg} \mathrm{BW} 0.6 / \mathrm{d})=[\mathrm{N}$ intake $(\mathrm{g})$ - fecal $\mathrm{N}$ output $(\mathrm{g})$ - urine $\mathrm{N}$ output $(\mathrm{g})] \times 6.25 \times 23.86(\mathrm{~kJ} / \mathrm{g}) / \mathrm{BW}$.

${ }^{6} \mathrm{RE}_{\mathrm{L}}=$ Energy retention as fat $\left(\mathrm{kJ} / \mathrm{kg} \mathrm{BW}{ }^{0.6} / \mathrm{d}\right)=[\mathrm{RE}(\mathrm{kJ})$ - energy retention as protein $(\mathrm{kJ})] / \mathrm{BW}^{0.6}$.

${ }^{7}$ Linear and Quadratic represents the polynomial contrasts analysis on linear and quadratic effects of the ambient temperatures. 


\begin{tabular}{|c|c|c|c|c|c|c|c|c|c|c|}
\hline \multirow[t]{2}{*}{ Items } & \multicolumn{6}{|c|}{ Ambient temperature $\left({ }^{\circ} \mathrm{C}\right)$} & \multirow[t]{2}{*}{ SEM } & \multicolumn{3}{|c|}{ P-value ${ }^{7}$} \\
\hline & 18 & 21 & 23 & 27 & 29 & 32 & & ANOVA & Linear & Quadratic \\
\hline $\mathrm{RE}_{\mathrm{P}}{ }^{5}$ & $594^{\mathrm{a}}$ & $558^{\mathrm{ab}}$ & $602^{\mathrm{a}}$ & $491^{\mathrm{ab}}$ & $521^{\mathrm{ab}}$ & $431^{\mathrm{b}}$ & 32.8 & 0.013 & $\hat{0.001}$ & 0.001 \\
\hline $\mathrm{RE}_{\mathrm{L}} 6$ & 971 & 860 & 879 & 715 & 957 & 714 & 121.0 & 0.409 & 0.220 & 0.471 \\
\hline \multicolumn{11}{|l|}{ Respiratory quotient } \\
\hline Fed state & 1.07 & 1.09 & 1.09 & 1.08 & 1.05 & 1.12 & 0.03 & 0.576 & 0.520 & 0.635 \\
\hline Fast state & $0.80^{\mathrm{b}}$ & $0.80^{\mathrm{b}}$ & $0.80^{\mathrm{b}}$ & $0.83^{\mathrm{ab}}$ & $0.83^{\mathrm{ab}}$ & $0.87^{a}$ & 0.02 & 0.025 & 0.003 & 0.007 \\
\hline \multicolumn{11}{|l|}{ Energy utilization, \% } \\
\hline $\mathrm{DE} / \mathrm{GE}$ & 84.9 & 84.3 & 84.9 & 86.7 & 87.6 & 84.9 & 1.13 & 0.293 & 0.211 & 0.307 \\
\hline Urine energy/DE & 1.0 & 1.1 & 1.1 & 1.2 & 1.4 & 1.3 & 0.18 & 0.709 & 0.114 & 0.283 \\
\hline Methane energy/DE & 0.35 & 0.42 & 0.38 & 0.50 & 0.40 & 0.43 & 0.07 & 0.829 & 0.426 & 0.559 \\
\hline ME/DE & 98.6 & 98.5 & 98.6 & 98.3 & 98.2 & 98.5 & 0.21 & 0.647 & 0.274 & 0.411 \\
\hline NE/ME & 85.1 & 85.4 & 83.4 & 84.1 & 88.7 & 86.0 & 2.30 & 0.577 & 0.441 & 0.709 \\
\hline RE/ME & 57.9 & 55.7 & 56.9 & 53.2 & 60.2 & 55.5 & 3.62 & 0.629 & 0.875 & 0.953 \\
\hline $\mathrm{RE}_{\mathrm{p}} / \mathrm{ME}$ & 22.0 & 22.2 & 23.6 & 22.2 & 21.5 & 22.1 & 0.68 & 0.347 & 0.522 & 0.513 \\
\hline $\mathrm{RE}_{\mathrm{L}} / \mathrm{ME}$ & 35.9 & 33.5 & 33.3 & 31.1 & 38.8 & 30.4 & 3.46 & 0.434 & 0.554 & 0.839 \\
\hline FHP/ME & 26.6 & 29.2 & 22.9 & 27.9 & 27.9 & 31.6 & 2.47 & 0.239 & 0.226 & 0.294 \\
\hline \multicolumn{11}{|c|}{ a,b Within a row means with different superscript letters differ at $P<0.05$. } \\
\hline \multicolumn{11}{|c|}{$\begin{array}{l}{ }^{1} \text { ANOVA, analysis of variance; DE, digestible energy; GE, gross energy; ME: metabolizable energy; NE: net energy; SEM, standard error } \\
\text { of the mean. }\end{array}$} \\
\hline \multicolumn{11}{|c|}{$\begin{array}{l}2 \text { Oxidation of carbohydrates }\left(\mathrm{kJ} / \mathrm{kg} \mathrm{BW} \mathrm{W}^{0.6} / \text { day }\right)=\left[-2.968 \times \mathrm{O}_{2}(\mathrm{~L})+4.147 \times \mathrm{CO}_{2}(\mathrm{~L})-1.761 \times \mathrm{CH}_{4}(\mathrm{~L})-2.446 \times \text { Urinary N }(\mathrm{g})\right] \times \\
17.58 / \mathrm{BW}^{0.6} \text {. }\end{array}$} \\
\hline \multicolumn{11}{|c|}{${ }^{3}$ Oxidation of fat $(\mathrm{kJ} / \mathrm{kg} \mathrm{BW} 0.6 /$ day $)=\left[1.719 \times \mathrm{O}_{2}(\mathrm{~L})-1.719 \times \mathrm{CO}_{2}(\mathrm{~L})-1.719 \times \mathrm{CH}_{4}(\mathrm{~L})-1.963 \times\right.$ Urinary N $\left.(\mathrm{g})\right] \times 39.76 / \mathrm{BW}^{0.6}$. } \\
\hline \multirow{2}{*}{\multicolumn{11}{|c|}{$\begin{array}{l}{ }^{4} \text { Total heat production adjusted }=\text { Total heat production was adjusted to the same ME intake at } 2.4 \mathrm{MJ} \mathrm{ME} / \mathrm{kg} \mathrm{BW} 0.6 / \mathrm{d} \text {. } \\
{ }^{5} \mathrm{RE}_{\mathrm{P}}=\text { Energy retention as protein }(\mathrm{kJ} / \mathrm{kg} \mathrm{BW} \cdot 0.6 / \mathrm{d})=[\mathrm{N} \text { intake }(\mathrm{g}) \text { - fecal } \mathrm{N} \text { output }(\mathrm{g}) \text { - urine N output }(\mathrm{g})] \times 6.25 \times 23.86(\mathrm{~kJ} / \mathrm{g}) / \mathrm{BW}^{0.6} \text {. }\end{array}$}} \\
\hline & & & & & & & & & & \\
\hline \multicolumn{11}{|c|}{${ }^{6} \mathrm{RE}_{\mathrm{L}}=$ Energy retention as fat $\left(\mathrm{kJ} / \mathrm{kg} \mathrm{BW} \mathrm{B}^{0.6} / \mathrm{d}\right)=[\mathrm{RE}(\mathrm{kJ})$ - energy retention as protein $(\mathrm{kJ})] / \mathrm{BW}^{0.6}$. } \\
\hline
\end{tabular}

\section{Energy partition in pigs at different BW under different ambient temperatures}

Comparison on energy partition of modern growing pigs at $25 \mathrm{~kg}$ and $65 \mathrm{~kg}$ stayed at $18{ }^{\circ} \mathrm{C}, 23^{\circ} \mathrm{C}, 27^{\circ} \mathrm{C}$, and $32^{\circ} \mathrm{C}$ were present in Table 3. There were significant interaction effects between $\mathrm{BW}$ and ambient temperature on $\mathrm{DMi}, \mathrm{N}$ balance (including $\mathrm{N}$ intake, fecal $\mathrm{N}$ output, urine $\mathrm{N}$ output and $\mathrm{N}$ retention), $\mathrm{ME}_{\mathrm{i}}$, THP adjusted to the same $\mathrm{ME} \mathrm{F}_{\mathrm{i}}, \mathrm{RE}, \mathrm{RE} \mathrm{P}_{\mathrm{P}}, \mathrm{RE} \mathrm{L}_{\mathrm{L}}$, $\mathrm{RQ}$ in fed state, NE/ME, RE/ME and $\mathrm{RE} \mathrm{L}_{\mathrm{L}} / \mathrm{ME}(P<$ 0.05). However, the $M E_{i}, T H P$ adjusted to the same $M E_{i}, R E, R E_{p}, R E_{L}, R E / M E$ and $R E_{L} / M E$ did not exhibit difference between pigs at $25 \mathrm{~kg}$ and those at $65 \mathrm{~kg}$, and the NE/ME did not show difference among pigs under $18^{\circ} \mathrm{C}, 23^{\circ} \mathrm{C}, 27^{\circ} \mathrm{C}$, and $32^{\circ} \mathrm{C}(P>0.05)$. 
Table 3

¿Comparison between barrows at $25 \mathrm{~kg}$ and $65 \mathrm{~kg}$ on nitrogen and energy balance kept at different ambient temperatures ${ }^{1}$.

\begin{tabular}{|c|c|c|c|c|c|c|c|c|c|c|}
\hline \multirow[t]{2}{*}{ Items } & \multicolumn{4}{|c|}{ Ambient temperature $\left({ }^{\circ} \mathrm{C}\right)$} & \multicolumn{2}{|c|}{$\begin{array}{l}\text { Body weight } \\
\text { (kg) }\end{array}$} & \multirow[t]{2}{*}{ SEM } & \multicolumn{3}{|c|}{ Pvalue ${ }^{7}$} \\
\hline & 18 & 23 & 27 & 32 & 25 & 65 & & BW & $\mathbf{T}$ & ${ }_{\mathrm{T}}^{\mathrm{BW} \times}$ \\
\hline Dry matter intake, kg/d & 1.81 & 1.75 & 1.39 & 1.11 & 1.14 & 1.88 & 0.08 & $<.001$ & $\begin{array}{l}< \\
0.001\end{array}$ & <. 001 \\
\hline \multicolumn{11}{|l|}{ Nitrogen balance, g/d } \\
\hline Intake & 57.3 & 55.4 & 44.0 & 35.2 & 36.4 & 59.6 & 2.44 & $<.001$ & $\hat{0} 001$ & $<.001$ \\
\hline Fecal output & 8.9 & 7.4 & 5.6 & 4.9 & 5.4 & 8.0 & 0.57 & $<.001$ & $<001$ & 0.011 \\
\hline Urine output & 13.3 & 7.7 & 8.6 & 5.8 & 4.6 & 13.0 & 1.72 & $<.001$ & 0.001 & 0.003 \\
\hline Retention & 34.6 & 38.0 & 29.8 & 24.5 & 26.3 & 37.2 & 1.92 & $\dot{0} 001$ & $\begin{array}{l}< \\
0.001\end{array}$ & 0.042 \\
\hline \multicolumn{11}{|l|}{ 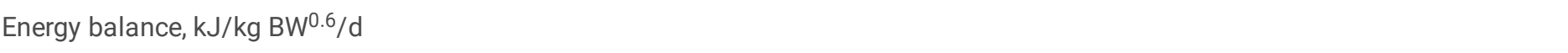 } \\
\hline ME intake & 2737 & 2924 & 2193 & 1751 & 2375 & 2427 & 119.7 & 0.514 & $\begin{array}{l}< \\
0.001\end{array}$ & <. 001 \\
\hline Total heat production & $1168^{a}$ & $1120^{\mathrm{a}}$ & $998^{b}$ & $894^{b}$ & 1024 & 1066 & 53.8 & 0.130 & $<.001$ & 0.601 \\
\hline $\begin{array}{l}\text { Oxidation of carbohydrates } \text { fed state } \\
2\end{array}$ & $1251^{a b}$ & $1483^{a}$ & $1162^{b}$ & $1013^{b}$ & 1320 & 1134 & 114.2 & 0.022 & 0.002 & 0.424 \\
\hline Oxidation of fat fed state ${ }^{3}$ & -274 & -399 & -305 & -140 & -310 & -249 & 97.8 & 0.397 & 0.104 & 0.438 \\
\hline Total heat production adjusted 4 & 1032 & 941 & 1111 & 1238 & 1057 & 1104 & 76.9 & 0.333 & 0.001 & 0.007 \\
\hline Fasting heat production (FHP) & 725 & 723 & 678 & 712 & 709 & 710 & 60.6 & 0.991 & 0.860 & 0.875 \\
\hline $\begin{array}{l}\text { Oxidation of carbohydrates fast } \\
\text { state }^{2}\end{array}$ & 150 & 210 & 201 & 258 & 252 & 157 & 44.2 & 0.001 & 0.056 & 0.149 \\
\hline Oxidation of fat fast state ${ }^{3}$ & 375 & 349 & 334 & 300 & 385 & 294 & 65.1 & 0.060 & 0.732 & 0.424 \\
\hline
\end{tabular}

a-c Within a row means with different superscript letters differ at $P<0.05$. Different means are separated with superscripts in the table to illustrate the significant main effects only when the interaction effect is not significant.

${ }^{1} \mathrm{DE}$, digestible energy; GE, gross energy; ME: metabolizable energy; NE: net energy; SEM, standard error of the mean.

2 Oxidation of carbohydrates $(\mathrm{kJ} / \mathrm{kg} \mathrm{BW} 0.6 /$ day $)=\left[-2.968 \times \mathrm{O}_{2}(\mathrm{~L})+4.147 \times \mathrm{CO}_{2}(\mathrm{~L})-1.761 \times \mathrm{CH}_{4}(\mathrm{~L})-2.446 \times \mathrm{Urinary} \mathrm{N}(\mathrm{g})\right] \times$ $17.58 / \mathrm{BW}^{0.6}$

${ }^{3}$ Oxidation of fat $\left(\mathrm{kJ} / \mathrm{kg} \mathrm{BW} \mathrm{B}^{0.6} /\right.$ day $\left.)=\left[1.719 \times \mathrm{O}_{2}(\mathrm{~L})-1.719 \times \mathrm{CO}_{2}(\mathrm{~L})-1.719 \times \mathrm{CH}_{4}(\mathrm{~L})-1.963 \times \mathrm{Urinary} \mathrm{N}^{\mathrm{g}} \mathrm{g}\right)\right] \times 39.76 / \mathrm{BW}^{0.6}$.

${ }^{4}$ Total heat production adjusted = Total heat production was adjusted to the same ME intake at $2.4 \mathrm{MJ} \mathrm{ME} / \mathrm{kg} \mathrm{BW}^{0.6} / \mathrm{d}$.

${ }^{5} \mathrm{RE}_{\mathrm{P}}=$ Energy retention as protein $(\mathrm{kJ} / \mathrm{kg} \mathrm{BW} 0.6 / \mathrm{d})=[\mathrm{N}$ intake $(\mathrm{g})-$ fecal $\mathrm{N}$ output $(\mathrm{g})-$ urine $\mathrm{N}$ output $(\mathrm{g})] \times 6.25 \times 23.86(\mathrm{~kJ} / \mathrm{g}) / \mathrm{BW}$. 6.

${ }^{6} \mathrm{RE}_{\mathrm{L}}=$ Energy retention as fat $\left(\mathrm{kJ} / \mathrm{kg} \mathrm{BW} \mathrm{B}^{0.6} / \mathrm{d}\right)=[\mathrm{RE}(\mathrm{kJ})$ - energy retention as protein $(\mathrm{kJ})] / \mathrm{BW}^{0.6}$.

${ }^{7} \mathrm{BW}, \mathrm{T}$, and BW $\times$ T represents the main effects of body weight, the main effect of ambient temperature, and the interaction effect between body weight and ambient temperature, respectively. 


\begin{tabular}{|c|c|c|c|c|c|c|c|c|c|c|}
\hline \multirow[t]{2}{*}{ Items } & \multicolumn{4}{|c|}{ Ambient temperature $\left({ }^{\circ} \mathrm{C}\right)$} & \multicolumn{2}{|c|}{$\begin{array}{l}\text { Body weight } \\
\text { (kg) }\end{array}$} & \multirow[t]{2}{*}{ SEM } & \multicolumn{3}{|c|}{$P_{\text {-value }}{ }^{7}$} \\
\hline & 18 & 23 & 27 & 32 & 25 & 65 & & BW & $\mathrm{T}$ & $\mathrm{BW}_{\mathrm{T}}$ \\
\hline Energy retention (RE) & 1569 & 1804 & 1195 & 857 & 1351 & 1361 & 124.0 & 0.896 & $\dot{0} 001$ & $\hat{0} 001$ \\
\hline $\mathrm{RE}_{\mathrm{P}} 5$ & 581 & 663 & 469 & 364 & 528 & 511 & 37.1 & 0.517 & $\dot{0} 001$ & 0.015 \\
\hline $\mathrm{RE}_{\mathrm{L}}{ }^{6}$ & 988 & 1141 & 724 & 540 & 822 & 874 & 101.9 & 0.437 & $\dot{0} 001$ & <. 0.001 \\
\hline \multicolumn{11}{|l|}{ Respiratory quotient } \\
\hline Fed state & 1.08 & 1.09 & 1.07 & 1.05 & 1.08 & 1.06 & 0.02 & 0.059 & 0.140 & 0.013 \\
\hline Fast state & 0.80 & 0.80 & 0.81 & 0.84 & 0.82 & 0.81 & 0.02 & 0.496 & 0.084 & 0.893 \\
\hline \multicolumn{11}{|l|}{ Energy utilization, \% } \\
\hline $\mathrm{DE} / \mathrm{GE}$ & 85.7 & 87.1 & 87.8 & 86.5 & 85.7 & 87.8 & 0.91 & 0.003 & 0.171 & 0.230 \\
\hline Urine energy/DE & 1.3 & 1.1 & 1.4 & 1.6 & 1.1 & 1.6 & 0.24 & 0.006 & 0.245 & 0.158 \\
\hline Methane energy/DE & $0.41^{\mathrm{b}}$ & $0.42^{\mathrm{ab}}$ & $0.63^{\mathrm{ab}}$ & $0.64^{\mathrm{a}}$ & 0.42 & 0.63 & 0.08 & 0.002 & 0.011 & 0.188 \\
\hline ME/DE & 98.3 & 98.4 & 97.9 & 97.8 & 98.4 & 97.8 & 0.30 & 0.006 & 0.228 & 0.112 \\
\hline NE/ME & 78.5 & 81.6 & 78.5 & 76.3 & 84.8 & 72.7 & 2.00 & $\hat{0} .001$ & 0.084 & 0.006 \\
\hline RE/ME & 57.0 & 60.8 & 53.7 & 48.4 & 56.0 & 54.0 & 3.20 & 0.333 & 0.001 & 0.007 \\
\hline $\mathrm{RE}_{\mathrm{p}} / \mathrm{ME}$ & 21.2 & 22.8 & 22.2 & 21.3 & 22.3 & 21.5 & 0.81 & 0.154 & 0.139 & 0.534 \\
\hline $\mathrm{RE}_{\mathrm{L}} / \mathrm{ME}$ & 35.8 & 38.0 & 32.2 & 29.3 & 33.7 & 34.0 & 3.32 & 0.881 & 0.037 & 0.031 \\
\hline FHP/ME & $26.8^{\mathrm{bc}}$ & $22.6^{c}$ & $30.4^{\mathrm{b}}$ & $39.4^{\mathrm{a}}$ & 27.8 & 31.8 & 3.17 & 0.049 & $\hat{0.001}$ & 0.259 \\
\hline \multicolumn{11}{|c|}{$\begin{array}{l}\text { a-c Within a row means with different superscript letters differ at } P<0.05 \text {. Different means are separated with superscripts in the table } \\
\text { to illustrate the significant main effects only when the interaction effect is not significant. }\end{array}$} \\
\hline \multicolumn{11}{|c|}{${ }^{1} \mathrm{DE}$, digestible energy; GE, gross energy; ME: metabolizable energy; NE: net energy; SEM, standard error of the mean. } \\
\hline \multicolumn{11}{|c|}{$\begin{array}{l}2 \text { Oxidation of carbohydrates }(\mathrm{kJ} / \mathrm{kg} \mathrm{BW} 0.6 / \text { day })=\left[-2.968 \times \mathrm{O}_{2}(\mathrm{~L})+4.147 \times \mathrm{CO}_{2}(\mathrm{~L})-1.761 \times \mathrm{CH}_{4}(\mathrm{~L})-2.446 \times \text { Urinary N }(\mathrm{g})\right] \times \\
17.58 / \mathrm{BW}^{0.6} \text {. }\end{array}$} \\
\hline \multicolumn{11}{|c|}{${ }^{3}$ Oxidation of fat $(\mathrm{kJ} / \mathrm{kg} \mathrm{BW} 0.6 /$ day $)=\left[1.719 \times \mathrm{O}_{2}(\mathrm{~L})-1.719 \times \mathrm{CO}_{2}(\mathrm{~L})-1.719 \times \mathrm{CH}_{4}(\mathrm{~L})-1.963 \times\right.$ Urinary N $\left.(\mathrm{g})\right] \times 39.76 / \mathrm{BW}^{0.6}$. } \\
\hline \multicolumn{11}{|c|}{${ }^{4}$ Total heat production adjusted = Total heat production was adjusted to the same ME intake at $2.4 \mathrm{MJ} \mathrm{ME} / \mathrm{kg} \mathrm{BW} 0.6 / \mathrm{d}$. } \\
\hline \multicolumn{11}{|c|}{${ }^{5} \mathrm{RE}_{\mathrm{p}}=$ Energy retention as protein $\left(\mathrm{kJ} / \mathrm{kg} \mathrm{BW} \mathrm{W}^{0.6} / \mathrm{d}\right)=[\mathrm{N}$ intake $(\mathrm{g})$ - fecal $\mathrm{N}$ output $(\mathrm{g})$ - urine $\mathrm{N}$ output $(\mathrm{g})] \times 6.25 \times 23.86(\mathrm{~kJ} / \mathrm{g}) / \mathrm{BW}^{0.6}$. } \\
\hline \multicolumn{11}{|c|}{${ }^{6} \mathrm{RE}_{\mathrm{L}}=$ Energy retention as fat $(\mathrm{kJ} / \mathrm{kg} \mathrm{BW} 0.6 / \mathrm{d})=[\mathrm{RE}(\mathrm{kJ})$ - energy retention as protein $(\mathrm{kJ})] / \mathrm{BW}^{0.6}$. } \\
\hline
\end{tabular}

For the other parameters without interaction effects, pigs at $65 \mathrm{~kg}$ showed greater DE/GE, urine energy/DE, methane energy/DE and $\mathrm{FHP} / \mathrm{ME}$, but lower $\mathrm{OXCHO}$ at both fed state and fast state, and ME/DE compared with pigs at $25 \mathrm{~kg}(P<0.05)$. In addition, pigs stayed at $27^{\circ} \mathrm{C}$ and $32{ }^{\circ} \mathrm{C}$ demonstrated lower THP and lower OXCHO at fed state than pigs stayed at $18^{\circ} \mathrm{C}$ and $23^{\circ} \mathrm{C}$, and pigs stayed at $23^{\circ} \mathrm{C}$, respectively; while pigs stayed at $32^{\circ} \mathrm{C}$ exhibited greater methane energy/DE and greater FHP/ME than pigs stayed at $18{ }^{\circ} \mathrm{C}$, and pigs stayed under the other three ambient temperatures $(P<0.05)$. 


\section{Modelling the voluntary feed intake and energy partition considering pigs' BW and ambient temperature}

The quadratic effects of BW and T as well as their interaction effect on response variables were further confirmed. Models developed in the current study to predict $\mathrm{VFI}, \mathrm{ME}_{\mathrm{i}}, \mathrm{RE}_{\mathrm{P}}$ and $\mathrm{RE} \mathrm{L}_{\mathrm{L}}$ using both $\mathrm{BW}$ and ambient temperature $(\mathrm{T})$ as predictors were:

$\operatorname{VFI}(\mathrm{kg} / \mathrm{d})=-7.72+1.54 \times \mathrm{BW}^{0.6}-0.059 \times\left(B W^{0.6}\right)^{2}+0.15 \times \mathrm{T}-0.0022 \times \mathrm{T}^{2}-0.0088 \times B W^{0.6} \times \mathrm{T}^{2}\left(\mathrm{R}^{2}=0.86\right)$

$\mathrm{ME}_{\mathrm{i}}\left(\mathrm{kJ} / \mathrm{kg} \mathrm{BW} \mathrm{W}^{0.6} / \mathrm{d}\right)=-9242.45+2042.05 \times B W^{0.6}-91.95 \times\left(B W^{0.6}\right)^{2}+242.32 \times \mathrm{T}-4.56 \times \mathrm{T}^{2}-8.33 \times B W^{0.6} \times \mathrm{T}^{2}\left(\mathrm{R}^{2}=0.67\right)$

$R E_{P}\left(k J / k g B W^{0.6} / d\right)=-233.38-67.28 \times B W^{0.6}+3.16 \times\left(B W^{0.6}\right)^{2}+39.58 \times T-0.76 \times T^{2}-0.26 \times B W^{0.6} \times T+0.26 \times M E_{i}\left(R^{2}=0.86\right) ;$

$\mathrm{RE}_{\mathrm{L}}\left(\mathrm{kJ} / \mathrm{kg} \mathrm{BW} \mathrm{W}^{0.6} / \mathrm{d}\right)=-465.10+8.10 \times B W^{0.6}-1.03 \times\left(B W^{0.6}\right)^{2}-53.73 \times \mathrm{T}+1.34 \times \mathrm{T}^{2}+0.67 \times \mathrm{BW}^{0.6} \times \mathrm{T}^{2}+0.69 \times \mathrm{ME}_{\mathrm{i}}\left(\mathrm{R}^{2}=0.85\right)$

which were present in Table 4. The estimated $\mathrm{VFI}, \mathrm{ME}_{\mathrm{i}}, \mathrm{RE}_{\mathrm{P}}$ and $\mathrm{RE} \mathrm{E}_{\mathrm{L}}$ of pigs at $25 \mathrm{~kg}$ and $65 \mathrm{~kg}$ under ambient temperatures from $18{ }^{\circ} \mathrm{C}$ to $32{ }^{\circ} \mathrm{C}$ based on these models were plotted in Fig. $1 \mathrm{~A}-\mathrm{D}$, which illustrated that feed intake and energy retention in pigs at $65 \mathrm{~kg}$ were more easily impaired by high temperatures than in pigs at $25 \mathrm{~kg}$. Meanwhile, the models for VFI and $\mathrm{ME}_{\mathrm{i}}$ were compared with the below models developed previously (Fig. 1A and 1B):

Table 4

पPrediction equations for voluntary feed intake, metabolizable energy intake, energy retention as protein and energy retention as lipid using body weight and ambient temperature as predictors.

\begin{tabular}{|c|c|c|c|c|c|c|c|c|c|c|c|}
\hline \multirow[t]{2}{*}{ No. } & \multirow[t]{2}{*}{ Models } & \multicolumn{7}{|c|}{ Coefficients estimation 2} & \multirow[t]{2}{*}{ RMSE } & \multirow[t]{2}{*}{$\mathbf{R}^{2}$} & \multirow{2}{*}{$\begin{array}{l}P \\
\text { value }\end{array}$} \\
\hline & & a & b & c & d & e & $\mathbf{f}$ & $\mathbf{g}$ & & & \\
\hline 1 & $\begin{array}{l}\mathrm{VFI}=\mathrm{a}+\mathrm{b} \times \mathrm{BW}^{0.6}+\mathrm{c} \times \\
\left(B W^{0.6}\right)^{2}+ \\
\mathrm{d} \times \mathrm{T}+\mathrm{e} \times \mathrm{T}^{2}+\mathrm{f} \times \mathrm{BW}^{0.6} \times\end{array}$ & -7.72 & 1.54 & -0.059 & 0.15 & -0.0022 & -0.0088 & / & 0.18 & 0.86 & $\begin{array}{l}< \\
0.001\end{array}$ \\
\hline 2 & $\begin{array}{l}M_{i}=a+b \times B W^{0.6}+c \times \\
\left(B W^{0.6}\right)^{2}+ \\
d \times T+e \times T^{2}+f \times B W^{0.6} \times \\
d T\end{array}$ & -9242.45 & 2042.05 & -91.95 & 242.32 & -4.56 & -8.33 & / & 316.30 & 0.67 & $\begin{array}{l}< \\
0.001\end{array}$ \\
\hline 3 & $\begin{array}{l}\mathrm{RE}_{P}=\mathrm{a}+\mathrm{b} \times \mathrm{BW}^{0.6}+\mathrm{c} \times \\
\left(B W^{0.6}\right)^{2}+ \\
\mathrm{d} \times \mathrm{T}+\mathrm{e} \times \mathrm{T}^{2}+\mathrm{f} \times \mathrm{BW}^{0.6} \times \\
\mathrm{T}+\mathrm{g} \times \mathrm{ME}_{\mathrm{i}}\end{array}$ & -233.38 & -67.28 & 3.16 & 39.58 & -0.76 & -0.26 & 0.26 & 61.66 & 0.86 & $\begin{array}{l}< \\
0.001\end{array}$ \\
\hline 4 & $\begin{array}{l}\mathrm{RE}_{\mathrm{L}}=\mathrm{a}+\mathrm{b} \times \mathrm{BW}^{0.6}+\mathrm{c} \times \\
\left(\mathrm{BW}^{0.6}\right)^{2}+ \\
\mathrm{d} \times \mathrm{T}+\mathrm{e} \times \mathrm{T}^{2}+\mathrm{f} \times \mathrm{BW}^{0.6} \times \\
\mathrm{T}+\mathrm{g} \times \mathrm{ME}_{\mathrm{i}}\end{array}$ & -465.10 & 8.10 & -1.03 & -53.73 & 1.34 & 0.67 & 0.69 & 134.58 & 0.85 & $\begin{array}{l}< \\
0.001\end{array}$ \\
\hline $\begin{array}{l}{ }^{1} \mathrm{BV} \\
\text { reter }\end{array}$ & $\begin{array}{l}\text { ody weight }(\mathrm{kg}) ; \mathrm{ME}_{\mathrm{i}} \text {, meta } \\
\text { on as protein }\left(\mathrm{kJ} / \mathrm{kg} \mathrm{BW} \mathrm{BW}^{0.6}\right.\end{array}$ & $\begin{array}{l}\text { izable ener } \\
\text { RMSE, roo }\end{array}$ & $\begin{array}{l}\text { intake } \\
\text { nean sq }\end{array}$ & $\begin{array}{l}\text { l } \mathrm{kg} \mathrm{BW} \\
\text { e error; }\end{array}$ & $\begin{array}{l}\text { (d); } \mathrm{RE}_{\mathrm{L}} \\
\text { ambien }\end{array}$ & $\begin{array}{l}\text { iergy ret } \\
\text { mperatc }\end{array}$ & $\begin{array}{l}\text { ion as I } \\
\left({ }^{\circ} \mathrm{C}\right) \text {; VF }\end{array}$ & $\begin{array}{l}\mathrm{d}(\mathrm{kJ} / \\
\text { /olunt }\end{array}$ & $\begin{array}{l}\mathrm{BW}^{0.6} / \mathrm{C} \\
\text { feed in }\end{array}$ & $\begin{array}{l}\mathrm{RE}_{\mathrm{p}} \\
\mathrm{ke}(\mathrm{kg}\end{array}$ & ergy \\
\hline
\end{tabular}

VFI $(\mathrm{kg} / \mathrm{d})=-1.264+0.0736 \times \mathrm{BW}-0.00026 \times \mathrm{BW}^{2}+0.117 \times \mathrm{T}-0.0024 \times \mathrm{T}^{2}-0.00095 \times \mathrm{BW} \times \mathrm{T}$ (from Quiniou et al. [20]);

$\mathrm{ME}_{\mathrm{i}}(\mathrm{kJ} / \mathrm{d})=-26811.2+1268.4 \times \mathrm{BW}-5.215 \times \mathrm{BW}^{2}+1572.3 \times \mathrm{T}-27.48 \times \mathrm{T}^{2}-15.7 \times \mathrm{BW} \times \mathrm{T}$ (from Guo [21]); 
Nearly parallel VFI curves were present between models in the current study and those built previously, but narrower gaps between $\mathrm{ME}_{\mathrm{i}}$ curves representing different BWs were found in our study compared with those from previous study especially under high ambient temperatures. Moreover, the goodness-of-fit of those developed models were shown in Fig. 2 using predicted vs. actual plot and predicted vs. residuals plot, indicating no problems on fitness of the models developed in the current study.

Changes in hormone and biochemical markers levels in serum of pigs at $25 \mathrm{~kg}$ under different ambient temperatures

The concentrations of hormone and biomedical markers in serum of modern growing pigs at $25 \mathrm{~kg}$ kept at $18{ }^{\circ} \mathrm{C}, 21^{\circ} \mathrm{C}, 23^{\circ} \mathrm{C}, 27^{\circ} \mathrm{C}, 29^{\circ} \mathrm{C}$ and $32{ }^{\circ} \mathrm{C}$ were present in Table 5. The levels of cortisol, $\mathrm{T}_{3}, \mathrm{~T}_{4}, \mathrm{HDL}$ and TC in pig serum showed patterns of linearly and quadratically decreased as the ambient temperature increased from $18{ }^{\circ} \mathrm{C}$ to $32^{\circ} \mathrm{C}$, with the $\mathrm{T}_{4}$ level at $18^{\circ} \mathrm{C}$ greater than that at $27^{\circ} \mathrm{C}, 29^{\circ} \mathrm{C}$ and $32{ }^{\circ} \mathrm{C}$, the HDL level at $18^{\circ} \mathrm{C}$ greater than that at $27^{\circ} \mathrm{C}$ and $29^{\circ} \mathrm{C}$, and the TC level at $18^{\circ} \mathrm{C}, 21^{\circ} \mathrm{C}$ and $23^{\circ} \mathrm{C}$ greater than that at $27^{\circ} \mathrm{C}(P<0.05)$. Moreover, the glucagon and AST levels in pig serum quadratically changed as the ambient temperature increased from $18^{\circ} \mathrm{C}$ to $32^{\circ} \mathrm{C}$, with the AST level at $29^{\circ} \mathrm{C}$ greater than that at $18^{\circ} \mathrm{C}(P<0.05)$, and the glucagon level at $27^{\circ} \mathrm{C}$ tended to be the lowest among all the treatment groups $(P=0.054)$.

Table 5

पHormone and biochemical markers levels in serum of barrows at $25 \mathrm{~kg}$ kept at different ambient temperature ${ }^{1}$.

\begin{tabular}{|c|c|c|c|c|c|c|c|c|c|c|}
\hline \multirow[t]{2}{*}{ Items } & \multicolumn{6}{|c|}{ Ambient temperature $\left({ }^{\circ} \mathrm{C}\right)$} & \multirow[t]{2}{*}{ SEM } & \multicolumn{3}{|c|}{$P$-value ${ }^{2}$} \\
\hline & 18 & 21 & 23 & 27 & 29 & 32 & & ANOVA & Linear & Quadratic \\
\hline Cortisol, ng/ml & 61.8 & 67.3 & 61.6 & 59.2 & 47.3 & 36.9 & 7.87 & 0.058 & 0.006 & 0.010 \\
\hline Glucagon, $\mathrm{pg} / \mathrm{ml}$ & 89.6 & 83.9 & 76.6 & 63.6 & 83.6 & 98.1 & 6.73 & 0.054 & 0.673 & 0.012 \\
\hline Growth hormone, ng/ml & 4.53 & 5.23 & 6.56 & 4.06 & 4.97 & 5.97 & 0.98 & 0.571 & 0.707 & 0.930 \\
\hline Insulin, $\mu \mathrm{lU} / \mathrm{ml}$ & 8.69 & 8.00 & 8.68 & 7.59 & 7.22 & 8.09 & 0.54 & 0.470 & 0.171 & 0.335 \\
\hline Thyroxine, $\mathrm{ng} / \mathrm{ml}$ & $58.6^{\mathrm{a}}$ & $46.2^{\mathrm{ab}}$ & $45.7^{\mathrm{ab}}$ & $41.3^{b c}$ & $33.6^{\mathrm{bc}}$ & $31.0^{\mathrm{C}}$ & 3.66 & $<0.001$ & $<0.001$ & $<0.001$ \\
\hline Triiodothyronine, ng/ml & 0.63 & 0.62 & 0.59 & 0.55 & 0.59 & 0.52 & 0.03 & 0.061 & 0.010 & 0.039 \\
\hline Albumin, $\mu \mathrm{mol} / \mathrm{L}$ & 35.7 & 35.8 & 35.4 & 33.8 & 35.0 & 37.2 & 0.98 & 0.303 & 0.661 & 0.212 \\
\hline ALT, U/L & 61.2 & 53.8 & 60.4 & 50.5 & 55.5 & 56.0 & 3.34 & 0.337 & 0.244 & 0.309 \\
\hline AST, U/L & $35.2^{\mathrm{b}}$ & $36.8^{\mathrm{ab}}$ & $48.5^{\mathrm{ab}}$ & $54.3^{\mathrm{ab}}$ & $55.5^{\mathrm{a}}$ & $39.2^{\mathrm{ab}}$ & 4.62 & 0.013 & 0.082 & 0.005 \\
\hline Globulin, $\mu \mathrm{mol} / \mathrm{L}$ & 34.1 & 31.6 & 37.2 & 35.1 & 34.9 & 33.0 & 1.27 & 0.128 & 0.937 & 0.438 \\
\hline $\mathrm{HDL}, \mathrm{mmol} / \mathrm{L}$ & $0.55^{\mathrm{a}}$ & $0.49^{a b c}$ & $0.53^{\mathrm{ab}}$ & $0.40^{\mathrm{c}}$ & $0.43^{b c}$ & $0.46^{\mathrm{abc}}$ & 0.03 & 0.004 & 0.004 & 0.006 \\
\hline LDL, mmol/L & 1.55 & 1.36 & 1.60 & 1.34 & 1.45 & 1.32 & 0.07 & 0.052 & 0.064 & 0.168 \\
\hline Total cholesterol, mmol/L & $2.47^{\mathrm{a}}$ & $2.30^{\mathrm{a}}$ & $2.40^{\mathrm{a}}$ & $2.05^{b}$ & $2.25^{\mathrm{ab}}$ & $2.26^{\mathrm{ab}}$ & 0.05 & 0.002 & 0.017 & 0.007 \\
\hline Triglyceride, $\mathrm{mmol} / \mathrm{L}$ & 0.54 & 0.44 & 0.48 & 0.54 & 0.49 & 0.40 & 0.04 & 0.134 & 0.198 & 0.351 \\
\hline Total protein, $\mathrm{g} / \mathrm{L}$ & 69.8 & 67.4 & 68.8 & 71.5 & 69.1 & 70.2 & 2.06 & 0.845 & 0.556 & 0.834 \\
\hline Urea, mmol/L & 4.21 & 3.84 & 4.50 & 3.76 & 3.99 & 4.23 & 0.18 & 0.306 & 0.863 & 0.380 \\
\hline
\end{tabular}

a-c Within a row means with different superscript letters differ at $P<0.05$.

${ }^{1}$ ALT, glutamic-pyruvic transaminase; AST, glutamic oxalacetic transaminase; ANOVA, analysis of variance; HDL, high density lipoprotein; LDL, low density lipoprotein; SEM, standard error of the mean.

${ }^{2}$ Linear and Quadratic represents the polynomial contrasts analysis on linear and quadratic effects of the ambient temperatures.

Metabolomics profiles in plasma of pigs at $25 \mathrm{~kg}$ under different ambient temperatures

The PCA score plot showed that the plasma samples from pigs stayed at low $\left(18^{\circ} \mathrm{C}\right)$, neutral $\left(23^{\circ} \mathrm{C}\right)$ and high $\left(32^{\circ} \mathrm{C}\right)$ ambient temperatures obviously clustered within the $95 \%$ confidence interval (Fig. 3). The PC1 and PC2 accounted for $41.9 \%$ and $9.98 \%$ of the total 
variance of the data, respectively, indicating there were major difference among the metabolomics profiles of the three treatment groups.

A total of 13 compounds were identified with different intensities among the three treatment groups by comparing to the standard entries in reference libraries (Table 6), which include categories of amino acid, fatty acid and steroid. To better illustrate the changes of those compounds in the treatment groups, data were visualized through boxplot in Fig. 4. Compared to pigs stayed at the neutral temperature $\left(23^{\circ} \mathrm{C}\right)$ or low temperature $\left(18^{\circ} \mathrm{C}\right)$, the concentrations of $\left(2^{\prime} \mathrm{E}, 4^{\prime} \mathrm{Z}, 7^{\prime} \mathrm{Z}, 8 \mathrm{E}\right)$-colnelenic acid, 3-beta-hydroxy-5-cholestenoate, adrenic acid, dihydrocortisol and indoleacetic acid all dramatically up-regulated, while the concentrations of 20-hydroxyeicosatetraenoic acid, betasitosterol, cortisol, deoxyuridine, leukotriene $\mathrm{C} 4$, phenylacetylglycine and spermidine all greatly down-regulated in plasma of pigs stayed at the high temperature $\left(32^{\circ} \mathrm{C}\right)$. In addition, compared to pigs stayed at neutral temperature, the levels of beta-sitosterol markedly upregulated, while the levels of ( $\left.2^{\prime} \mathrm{E}, 4^{\prime} Z, 7^{\prime} Z, 8 \mathrm{E}\right)$-colnelenic acid, cortisol, $L$-histidine, phenylacetylglycine and spermidine slightly downregulated in plasma of pigs stayed at the low temperature $\left(18^{\circ} \mathrm{C}\right)$.

Table 6

Metabolites with significantly different concentrations in plasma of barrows at $25 \mathrm{~kg}$ kept at different ambient temperatures.

\begin{tabular}{|c|c|c|c|c|c|c|c|}
\hline \multirow[t]{2}{*}{ No. } & \multirow[t]{2}{*}{ Compounds } & \multirow[t]{2}{*}{$\mathrm{m} / \mathrm{z}$} & \multirow[t]{2}{*}{ Formula } & \multicolumn{3}{|l|}{ Fold change $^{1}$} & \multirow[t]{2}{*}{ Pathway } \\
\hline & & & & High/Neutral & Low/Neutral & High/Low & \\
\hline 1 & $\begin{array}{l}(2 ' E, 4 ' Z, 7 ' Z, 8 E)- \\
\text { Colnelenic acid }\end{array}$ & 275.1994 & $\mathrm{C}_{18} \mathrm{H}_{28} \mathrm{O}_{3}$ & 4.97 & 0.84 & 5.91 & $\begin{array}{l}\text { alpha-Linolenic acid } \\
\text { metabolism }\end{array}$ \\
\hline 2 & $\begin{array}{l}20- \\
\text { Hydroxyeicosatetraenoic } \\
\text { acid }\end{array}$ & 303.2305 & $\mathrm{C}_{20} \mathrm{H}_{32} \mathrm{O}_{3}$ & 0.48 & 1.21 & 0.40 & $\begin{array}{l}\text { Arachidonic acid } \\
\text { metabolism }\end{array}$ \\
\hline 3 & $\begin{array}{l}\text { 3-beta-Hydroxy-5- } \\
\text { cholestenoate }\end{array}$ & 399.3242 & $\mathrm{C}_{27} \mathrm{H}_{44} \mathrm{O}_{3}$ & 2.39 & 1.22 & 1.96 & $\begin{array}{l}\text { Primary bile acid } \\
\text { biosynthesis }\end{array}$ \\
\hline 4 & Adrenic acid & 355.2614 & $\mathrm{C}_{22} \mathrm{H}_{36} \mathrm{O}_{2}$ & 4.83 & 1.11 & 4.35 & $\begin{array}{l}\text { Biosynthesis of } \\
\text { unsaturated fatty acids }\end{array}$ \\
\hline 5 & Beta-Sitosterol & 397.3812 & $\mathrm{C}_{29} \mathrm{H}_{50} \mathrm{O}$ & 0.24 & 2.36 & 0.10 & Steroid biosynthesis \\
\hline 6 & Cortisol & 363.2152 & $\mathrm{C}_{21} \mathrm{H}_{30} \mathrm{O}_{5}$ & 0.35 & 0.82 & 0.43 & $\begin{array}{l}\text { Steroid hormone } \\
\text { biosynthesis }\end{array}$ \\
\hline 7 & Deoxyuridine & 251.0629 & $\mathrm{C}_{9} \mathrm{H}_{12} \mathrm{~N}_{2} \mathrm{O}_{5}$ & 0.81 & 1.24 & 0.66 & Pyrimidine metabolism \\
\hline 8 & Dihydrocortisol & 382.2571 & $\mathrm{C}_{21} \mathrm{H}_{32} \mathrm{O}_{5}$ & 2.62 & 1.30 & 2.02 & $\begin{array}{l}\text { Steroid hormone } \\
\text { biosynthesis }\end{array}$ \\
\hline 9 & Indoleacetic acid & 176.0700 & $\mathrm{C}_{10} \mathrm{H}_{9} \mathrm{NO}_{2}$ & 2.61 & 1.01 & 2.59 & Tryptophan metabolism \\
\hline 10 & L-Histidine & 156.0763 & $\mathrm{C}_{6} \mathrm{H}_{9} \mathrm{~N}_{3} \mathrm{O}_{2}$ & 1.04 & 0.63 & 1.66 & $\begin{array}{l}\text { Histidine metabolism } \\
\text { /beta-Alanine } \\
\text { metabolism/Aminoacyl- } \\
\text { tRNA biosynthesis }\end{array}$ \\
\hline 11 & Leukotriene C4 & 313.6596 & $\mathrm{C}_{30} \mathrm{H}_{47} \mathrm{~N}_{3} \mathrm{O}_{9} \mathrm{~S}$ & 0.56 & 1.14 & 0.49 & $\begin{array}{l}\text { Arachidonic acid } \\
\text { metabolism }\end{array}$ \\
\hline 12 & Phenylacetylglycine & 211.1070 & $\mathrm{C}_{10} \mathrm{H}_{16} \mathrm{~N}_{2} \mathrm{O}_{4}$ & 0.57 & 0.79 & 0.73 & $\begin{array}{l}\text { Phenylalanine } \\
\text { metabolism }\end{array}$ \\
\hline 13 & Spermidine & 146.1647 & $\mathrm{C}_{7} \mathrm{H}_{19} \mathrm{~N}_{3}$ & 0.32 & 0.75 & 0.43 & $\begin{array}{l}\text { beta-Alanine } \\
\text { metabolism/Arginine } \\
\text { and proline metabolism } \\
\text { /Glutathione } \\
\text { metabolism }\end{array}$ \\
\hline
\end{tabular}

According to the pathway analysis, the identified metabolites are involved in pathways related to amino acid and lipid metabolism including histidine metabolism, beta-alanine metabolism, arachidonic acid metabolism, steroid hormone biosynthesis, and so on. However, only the pathway of histidine metabolism had an impact value greater than 0.1 (impact value $=0.22$ ), which is the cut-off value for relevance gained from the topology analysis. 


\section{Discussion}

\section{Effects of ambient temperature on voluntary feed intake and energy partition of modern growing pigs}

Most of the studies focusing on the VFI and energy partition patterns under different ambient temperatures in growing pigs were carried out in the second half of the 20th century. With the rapid developments in pig breeding, research updates on such topics concerning modern growing pigs have been absent in recent years.

Pig belongs to the warm-blooded animal. Under low ambient temperatures, the increased feed intake and heat production can facilitate the maintenance of a constant body temperature [22]. The criteria of "low" temperature is defined according to the thermoneutrual zone and the LCT value, which is usually estimated by BW. NRC [14] used the following equations to estimate the LCT in growing pigs: LCT $\left({ }^{\circ} \mathrm{C}\right)=$ 17.9-0.0375 $\times$ BW. Based on the temperature setting in the current study, all the pigs used in the animal trial were kept above the LCT. We observed an averaged $30 \mathrm{~g} / \mathrm{d} /{ }^{\circ} \mathrm{C}$ increase in $\mathrm{DMI}$ and $0.38 \mathrm{MJ} / \mathrm{d} /{ }^{\circ} \mathrm{C}$ increase in $\mathrm{ME}_{\mathrm{i}}$ when the ambient temperature decreased from $27^{\circ} \mathrm{C}$ to $18{ }^{\circ} \mathrm{C}$ for pigs with mean BW of $28 \mathrm{~kg}$ in the current study. Our results are similar to the VFI and energy intake patterns gained from Le Dividich et al. [23], who illustrated that the extra feed intake requirements were $32-33 \mathrm{~g} / \mathrm{d} /{ }^{\circ} \mathrm{C}$ and the additional DE required to compensate for $1{ }^{\circ} \mathrm{C}$ drop in temperature between $28^{\circ} \mathrm{C}$ and $20^{\circ} \mathrm{C}$ and between $20^{\circ} \mathrm{C}$ and $12{ }^{\circ} \mathrm{C}$ were 0.20 and $0.44 \mathrm{MJ} / \mathrm{d}$ in $30 \mathrm{~kg}$ growing pigs individually housed, respectively, but are different from the results from other studies. For example, Lefaucheur et al. [24] reported that an extra feed intake of $25 \mathrm{~g} / \mathrm{d} /{ }^{\circ} \mathrm{C}$ was required to achieve similar rates of weight gain for individual pig at 30 to $90 \mathrm{~kg}$ when environmental temperature decreased from $28{ }^{\circ} \mathrm{C}$ to $12{ }^{\circ} \mathrm{C}$. The extra feed intake requirements estimated by Quiniou et al. [1] were $19 \mathrm{~g} / \mathrm{d} /$ ${ }^{\circ} \mathrm{C}$ between $12{ }^{\circ} \mathrm{C}$ and $24^{\circ} \mathrm{C}$ for $30-90 \mathrm{~kg}$ pigs grouped-housed. Those different results may be attributed to the discrepancies in modern pig breeds and management conditions in pig farm nowadays, leading to more sensitive to the low environmental temperatures.

As the ambient temperature keeps raising, the heat production of the pig body becomes a "burden", and pigs can keep the body temperature fluctuate in a small range by increasing the evaporation heat loss and decreasing the heat production [22]. However, due to the degradation of the sweat gland, the capacity of heat dissipation through evaporation by pigs is limited [22]. Therefore, under high ambient temperatures, the feed intake of pigs will decrease dramatically to control their heat production [22]. For example, Collin et al. [25] showed that the VFI remained almost constant between $19{ }^{\circ} \mathrm{C}$ and $25^{\circ} \mathrm{C}$, and proportionally decreased $33 \mathrm{~g} /$ day $/{ }^{\circ} \mathrm{C}$ between $25^{\circ} \mathrm{C}$ and $35^{\circ} \mathrm{C}$ for pigs at $20 \mathrm{~kg}$. In the current study, we observed an averaged $56 \mathrm{~g} / \mathrm{d} /{ }^{\circ} \mathrm{C}$ in $\mathrm{DMl}$ and $0.90 \mathrm{MJ} / \mathrm{d} /{ }^{\circ} \mathrm{C}$ decrease in $\mathrm{ME}_{\mathrm{i}}$ when the ambient temperature increased from $29^{\circ} \mathrm{C}$ to $32{ }^{\circ} \mathrm{C}$ for pigs with mean BW of $28 \mathrm{~kg}$, but also observed an averaged $55 \mathrm{~g} / \mathrm{d} /{ }^{\circ} \mathrm{C}$ in $\mathrm{DMl}$ and $0.80 \mathrm{MJ} / \mathrm{d} /{ }^{\circ} \mathrm{C}$ increase in $\mathrm{ME}_{\mathrm{i}}$ when the ambient temperature increased from $27^{\circ} \mathrm{C}$ to $29^{\circ} \mathrm{C}$. The same pattern was also observed in THP adjusted into the same $\mathrm{ME}_{\mathrm{i}}$ level. Some previous studies also reported increased THP under high environmental temperatures, which could be attributed to the extra energy expenditure used for additional activity such as more frequent breathing and heart rating to facilitate the heat dissipation [9]. For instance, Close and Mount [26] illustrated that the THP was the lowest during $20^{\circ} \mathrm{C}$ to $25^{\circ} \mathrm{C}$, and gradually increased when the temperature reached $30^{\circ} \mathrm{C}$. The above conflict in heat production results may rely on the specific temperature range, BW and feeding level of the pigs [21]. Moreover, the increased consumption of dietary energy by pigs for heat dissipation in high temperature environment also leads to the decreased energy utilization efficiency.

The environmental temperature could affect the partition of $\mathrm{ME}_{\mathrm{i}}$, specifically, into maintenance energy, energy for thermoregulation, and energy for growth (the RE) in growing pigs. In the current study, no significant influence of ambient temperatures on maintenance energy $\left(\mathrm{ME}_{\mathrm{m}}\right.$, estimated by FHP), RE and the partial energy utilization efficiency, including partial efficiency for digestion (DE/GE), metabolization (ME/DE), net utilization (NE/ME), retention (RE/ME) and maintenance (FHP/ME), were observed. However, the ambient temperature altered the $\mathrm{OXCHO}$ and $\mathrm{RQ}$ during fast state as well as the $\mathrm{RE}_{\mathrm{P}}$, with $\mathrm{OXCHO}$ increased from $\sim 200$ to $\sim 400 \mathrm{~kJ} / \mathrm{kg} \mathrm{BW}^{0.75} / \mathrm{d}$, RE $\mathrm{P}_{\mathrm{P}}$ decreased from $\sim 600$ to $~ 430 \mathrm{~kJ} / \mathrm{kg} \mathrm{BW} 0.75 / \mathrm{d}$, and $\mathrm{RQ}$ increased from 0.80 to 0.87 , when environment temperature increased from $18^{\circ} \mathrm{C}$ to $32^{\circ} \mathrm{C}$, reflecting the enhanced maintenance energy partition at low ambient temperature and reduced protein synthesis at high ambient temperature. According to the previous study from Close [27], when the environmental temperature increased from $10{ }^{\circ} \mathrm{C}$ to $30^{\circ} \mathrm{C}$, the $\mathrm{ME}_{\mathrm{m}}$ decreased from 723 to $469 \mathrm{~kJ} / \mathrm{kg} \mathrm{BW}^{0.75} / \mathrm{d}$, and the RE efficiency (RE/ME) decreased from 0.79 to 0.63 , with a $\mathrm{ME}_{\mathrm{m}}$ of $440 \mathrm{~kJ} / \mathrm{kg}$ $\mathrm{BW}^{0.75} / \mathrm{d}$ and a RE efficiency of 0.67 at the thermoneutral temperature of $25^{\circ} \mathrm{C}$. In addition, both the partial energy efficiency estimated for protein deposition and lipid deposition were similar at each environmental temperature and decreased from 0.78 at $10{ }^{\circ} \mathrm{C}$ to 0.63 at $30{ }^{\circ} \mathrm{C}$ [27]. The above results are somehow in according with our observations, indicating that under low ambient temperature, more energy intake were used to maintain the body temperature of pigs, while part of energy was used to dissipate the heat produced during protein and lipid synthesis under high ambient temperature, leading to the decreased partial energy efficiency. Due to the absence of dietary

Page $15 / 22$ 
energy gradients settings, the changes of partial efficiency for protein and lipid synthesis under different temperatures were not tested in the current study.

\section{Effects of ambient temperature on nitrogen balance of modern growing pigs}

In the current study, we also focused on the effects of ambient temperatures on $\mathrm{N}$ balance. For pigs raised from $18{ }^{\circ} \mathrm{C}$ to $32^{\circ} \mathrm{C}$, the changing patterns of $\mathrm{N}$ intake and fecal $\mathrm{N}$ output were the same as that of the adjusted THP, which has been discussed previously. Moreover, the ratio of fecal $\mathrm{N}$ output to total $\mathrm{N}$ intake ranged from $12.5-16.7 \%$, and decreased from $18{ }^{\circ} \mathrm{C}$ to $29^{\circ} \mathrm{C}$, then increased from $29^{\circ} \mathrm{C}$ to $32^{\circ} \mathrm{C}$. This range exactly matched the range of $16-12 \%$ for fecal $\mathrm{N}$ output to total $\mathrm{N}$ intake ratio reported by Guo [21] when temperature changed from $23^{\circ} \mathrm{C}$ to $35^{\circ} \mathrm{C}$. Environmental temperature had no influence on urine $\mathrm{N}$ output in our study, which was also supported by Guo [21] and Verstegen et al. [3]. The ratio of $\mathrm{N}$ retention to total $\mathrm{N}$ intake ranged from $68.7 \%\left(32^{\circ} \mathrm{C}\right)$ to $76.0 \%\left(23^{\circ} \mathrm{C}\right)$, and is quite different from those of Verstegen et al. [3], who reported a range of $50 \%-54 \%$ of $\mathrm{N}$ retention to dietary $\mathrm{N}$ intake ratio, and was independent with ambient temperatures. Those discrepancies in results of $\mathrm{N}$ balance could be mainly ascribed to the differences in pig breeds and experimental conditions.

\section{Modelling the energy partition of modern growing pigs with different BW under different ambient temperatures}

It has been widely proved that pigs at different growth stages have distinct patterns of energy partition as the ambient temperatures change, since the critical temperatures differ for pigs with different BW [14]. In the current study, we found significant interaction effects between $\mathrm{BW}$ and temperature on $\mathrm{DMI}, \mathrm{N}$ balance, $\mathrm{ME}_{\mathrm{i}}$, adjusted THP, RE and the partial efficiency for lipid deposition (RE $/ \mathrm{ME}$ ), with pigs at $65 \mathrm{~kg}$ showed greater the above indexes except for $\mathrm{RE}_{\mathrm{p}}$ compared with pigs at $25 \mathrm{~kg}$. Therefore, the quadratic models including the main effects of metabolic BW, ambient temperature and their interaction effects were used to fit the raw data in the current study and to predict the $\mathrm{VFI}, \mathrm{ME}_{\mathrm{i}}$ as well as $\mathrm{RE}_{\mathrm{P}}$ and $\mathrm{RE}_{\mathrm{L}}$. Similar simulation equations have already been used to model the $\mathrm{VFI}$ [20], heat production and energy retention [1], and $\mathrm{ME}_{\mathrm{i}}$ [21] in pigs exposed to low or high ambient temperatures. Based on those curvilinear models developed in our study, it can be clearly see that pigs at heavier BW were more sensitive to high temperatures on $\mathrm{VFI} \mathrm{ME}_{\mathrm{i}}$ and protein and lipid deposition, which were partially supported by Renaudeau et al. [28], who analyzed the effects of high temperature on feed intake of pigs with different BW using meta-analysis based on 86 trials and 202 temperature treatments published from 1970 to 2009, and found that high temperature had a curvilinear effect on average daily feed intake, which was more pronounced in heavier pigs. In addition, the gaps between $\mathrm{ME}_{\mathrm{i}}$ curves representing different $\mathrm{BWs}$ in our study were narrower compared with those from previous studies using pigs with older genotypes, suggesting that modern genotypes could be more sensitive to heat stress than older genotypes of lesser growth potential. Similarly, Renaudeau et al. [28] also reported that the effect of increased temperature was greater in more contemporary publications. However, one flaw of the models developed in the current study is that the BW data collected for simulation only came from 2 growth stages, and the narrow range of BW may produce bias when predicting the responses on pigs with other BWs.

\section{Effects of ambient temperature on hormone and biochemical markers in serum of modern growing pigs}

As the ambient temperature increased from $18{ }^{\circ} \mathrm{C}$ to $32^{\circ} \mathrm{C}$, there was linearly decreased cortisol, $T_{3}, T_{4}, \mathrm{HDL}$ and TC levels, linearly increased AST level, and quadratically changed glucagon level in serum of $25 \mathrm{~kg}$ pigs in the current study. Similar changes in physiological and biomedical parameters in plasma of growing pigs under heat stress were also reported previously [29, 30]. The decreased cortisol content in plasma under low temperature may indicate improved protein degradation to provide amino acids for gluconeogenesis [30]. Moreover, the decreased cortisol level and increased glucagon and AST levels in plasma may also act as indicators for the stress reaction of pigs under extreme environmental conditions [30]. In addition, the depressed thyroid hormone $\left(T_{3}\right.$ and $\left.T_{4}\right)$ levels have been associated with lowered metabolic heat production in pigs kept at high environmental temperature [29]. Meanwhile, a reduction in HDL and TC, indicating the decreased $\beta$-oxidation of fat for energy purpose and favored hepatic synthesis of triglycerides, could also support the depressed plasma thyroid hormone levels in pigs reared at high ambient temperature [29].

\section{Effects of ambient temperature on plasma metabolomics profiles of modern growing pigs}

To simplify the analysis, only plasma samples from $18^{\circ} \mathrm{C}, 23^{\circ} \mathrm{C}$ and $32^{\circ} \mathrm{C}$ were used for metabolomics analysis in the current study. Among the 13 compounds identified, more compounds showed significant changes in pigs at high ambient temperature than those at low ambient temperature when compared to the thermoneutral condition, indicating that modern growing pigs are more sensitive to heat 
stress than cold stress reflected by the plasma metabolites. Another reason may be that the "low" temperature settings in the current study may not produce cold stress since it is above the LCT defined by NRC [14].

Under high ambient temperature, most of the metabolites with up-regulated expressions belong to fatty acids ((2'E,4'Z,7'Z,8E)-colnelenic acid, 3-beta-hydroxy-5-cholestenoate and adrenic acid), or involve in pathways related to lipid metabolism or fatty acid metabolism (dihydrocortisol), indicating elevated lipogenic pathways and suppressed fatty acid oxidation, which were also reflected by the levels of hormone and biochemical markers in serum in the current study, and was supported by the reports of Qu and Ajuwon [31], who showed greater serum linoleic and total polyunsaturated fatty acids levels in pigs stayed at $35^{\circ} \mathrm{C}$. On the other hand, the metabolites with downregulated expressions at $32^{\circ} \mathrm{C}$ are involved in amino acid metabolism (phenylacetylglycine and spermidine), or lipid metabolism (20hydroxyeicosatetraenoic acid, beta-sitosterol and leukotriene $\mathrm{C} 4$ ), which may indicate the enhanced protein degradation and gluconeogenesis, as well as the depressed lipolysis. Interestingly, cortisol was also identified in the metabolomics analysis, with downregulated level at high temperature, supporting the results in the plasma hormone assay. Furthermore, most of the metabolites in plasma only exhibited slightly changes in pigs at $18^{\circ} \mathrm{C}$ compared to that at $23^{\circ} \mathrm{C}$, suggesting less stress under this temperature setting.

\section{Conclusion}

The current study provided updated mathematical models to predict the voluntary feed intake and metabolizable energy intake as well as new models to estimate the energy requirements for protein and lipid deposition of growing pigs ( 25 to $65 \mathrm{~kg})$ under ambient temperatures of 18 to $32^{\circ} \mathrm{C}$. It also suggested that pigs at heavier body weight were more sensitive to high temperatures on energy intake and partition, and pigs with modern genotypes could be more sensitive to heat stress than pigs with older genotypes. The integrative analysis of indirect calorimetry and metabolomics profiling revealed that the decreased energy intake and total heat production under $32^{\circ} \mathrm{C}$ are associated with suppressed fatty acid oxidation and elevated lipogenesis and protein degradation, reflected by biomarkers including up-regulated adrenic acid and down-regulated cortisol levels in plasma. Our findings provide practical tools to precisely formulate diets for modern growing pigs under different ambient temperatures, and can help to improve our knowledge on potential mechanisms of thermoregulation in modern pig breeds.

\section{Abbreviations}

ALT, glutamic-pyruvic transaminase; AST, glutamic oxalacetic transaminase; BW, body weight; CP, crude protein; DE, digestible energy; DM, dry matter; DMi, dry matter intake; EE, ether extract; FHP, fasting heat production; GE, gross energy; GH, growth hormone; HDL, high density lipoprotein; HP, heat production; LCT, lower critical temperature; LDL, low density lipoprotein; $M E$, metabolizable energy; $M E_{i}$, metabolizable energy intake; $\mathrm{ME}_{\mathrm{m}}$ maintenance energy; $\mathrm{N}$, nitrogen; $\mathrm{NE}$, net energy; $\mathrm{OXCHO}$, oxidation of carbohydrates; $\mathrm{OXF}$, oxidation of fat; $\mathrm{PCA}$, principal components analysis; $R E$, energy retention; $R E_{L}$ energy retention as lipid; $R E_{p}$, energy retention as protein; $R Q$, respiratory quotient; $T_{3}$, triiodothyronine; $T_{4}$, thyroxine; $T C$, total cholesterol; TG, triglyceride; THP, total heat production; UCT, upper critical temperature; VFI, voluntary feed intake.

\section{Declarations}

\section{Availability of data and materials}

All the data were presented in the main manuscript and available to readers.

\section{Ethics approval}

All procedures used in this study were performed according to the guidelines for the ethical treatment of animal by the Institutional Animal Care and Use Committee of China Agricultural University (Beijing, China).

\section{Competing interests}

The authors declare no competing interests.

\section{Funding}


This work was supported by the National Key Research and Development Program of China (2016YFD0500506), the National Natural Science Foundation of China (31702121), and Sichuan Science and Technology Program (2018HH0160).

\section{Author's contributions}

SZ performed the data analysis and drafted the manuscript. HG carried out the experiment and produced all the data. XKY participated in the experiments. JJW critically evaluated the manuscript. JJZ designed the experiment and critically evaluated the manuscript. All authors read and approved the final manuscript.

\section{References}

1. Quiniou N, Noblet J, van Milgen J, Dubois S. Modelling heat production and energy balance in group-housed growing pigs exposed to low or high ambient temperatures. Br J Nutr. 2001;85(1):97-106.

2. Close W, Mount L, Start I. The influence of environmental temperature and plane of nutrition on heat losses from groups of growing pigs. Anim Sci. 1971;18(1):1-11.

3. Verstegen M, Close W, Start I, Mount L. The effects of environmental temperature and plane of nutrition on heat loss, energy retention and deposition of protein and fat in groups of growing pigs. Br J Nutr. 1973;30(1):21-35.

4. Dou S, Villa-Vialaneix N, Liaubet L, Billon Y, Giorgi M, Gilbert H, et al. 1HNMR-Based metabolomic profiling method to develop plasma biomarkers for sensitivity to chronic heat stress in growing pigs. PLoS One. 2017;12(11):e0188469.

5. Bruce J, Clark J. Models of heat production and critical temperature for growing pigs. Anim Prod. 1979;28:353-69.

6. Noblet J, Le Dividich J, Bikawa T. Interaction between energy level in the diet and environmental temperature on the utilization of energy in growing pigs. J Anim Sci. 1985;61(2):452-9.

7. van Milgen J, Quiniou N, Noblet J. Modelling the relation between energy intake and protein and lipid deposition in growing pigs. Anim Sci. 2000;71(1):119-30.

8. Collin A, van Milgen J, Dubois S, Noblet J. Effect of high temperature on feeding behaviour and heat production in group-housed young pigs. Br J Nutr. 2000;72(3):519-27.

9. Le Bellego L, van Milgen J, Noblet J. Effect of high ambient temperature on protein and lipid deposition and energy utilization in growing pigs. Anim Sci. 2002;75(1):85-96.

10. Stoddard E, Hovorka A. Animals, vulnerability and global environmental change: The case of farmed pigs in concentrated animal feeding operations in North Carolina. Geoforum. 2019;100, 153 - 65.

11. Li ZC, Liu H, Li YK, Lyu ZQ, Liu L, Lai CH, et al. Methodologies on estimating the energy requirements for maintenance and determining the net energy contents of feed ingredients in swine: A review of recent work. J Anim Sci Biotechnol. 2018;9:39.

12. Zhang GF, Liu DW, Wang FL, Li DF. Estimation of the net energy requirements for maintenance in growing and finishing pigs. J Anim Sci. 2014;92:2987-95.

13. Li EK, Liu H, Li YK, Liu L, Wang FL, Li DF, et al. Determination of net energy content of dietary lipids fed to growing pigs using indirect calorimetry. J Anim Sci. 2018;96:2184-94.

14. NRC. Nutrient requirements of swine. 11th ed. Washington: Nat Acad Press; 2012.

15. AOAC. Official methods of analysis. 18th ed. Arlington: Association of Official Chemists; 2007.

16. Brouwer E. Report of sub-committee on constants and factors. Proceedings of the 3rd EAAP Symposium on Energy Metabolism; Troonn, Publ. 11. London: Academic; 1965. p. 441-3.

17. Noblet J, Fortune H, Shi XS, Dubois S. Prediction of net energy value of feeds for growing pigs. J Anim Sci. 1994;72:344-54.

18. Chwalibog A, Jakobsen K, Henckel S, Thorbek G. Estimation of quantitative oxidation and fat retention from carbohydrate, protein and fat in growing pigs. J Anim Physiol Anim Nutr. 1992;68:123-35.

19. Liu H, Chen YF, Ming DX, Wang J, Li Z, Ma X, et al. Integrative analysis of indirect calorimetry and metabolomics profiling reveals alterations in energy metabolism between fed and fasted pigs. J Anim Sci Biotechnol. 2018;9:41.

20. Quiniou N, Dubois S, Noblet J. Voluntary feed intake and feeding behaviour of group-housed growing pigs are affected by ambient temperature and body weight. Livest Prod Sci. 2000;63(3):245-53.

21. Guo $\mathrm{CH}$. Modelling the effect of ambient temperature on protein and energy metabolism and utilization in growing-finishing pigs. Ph.D. dissertation. Sichuan Agricultural University, Chengdu, China. 2004.

Page $18 / 22$ 
22. Renaudeau D, Gilbert H, Noblet J. Effect of climatic environment on feed efficiency in swine. In: Patience JF, editor. Feed efficiency in swine. Wageningen: Wageningen Academic Publishers; 2012.

23. Le Dividich J, Noblet J, Bikawa T. Effect of environmental temperature and dietary energy concentration on the performance and carcass characteristics of growing-finishing pigs fed to equal rate of gain. Livest Prod Sci. 1987;17(3):235-46.

24. Lefaucheur L, Le Dividich J, Mourot J, Monin G, Ecolan P, Krauss D. Influence of environmental temperature on growth, muscle and adipose tissue metabolism, and meat quality in swine. J Anim Sci. 1991;69(7):2844-54.

25. Collin A, van Milgen J, Le Dividich J. Modelling the effect of high, constant temperature on food intake in young growing pigs. Anim Sci. 2001;72(3):519-27.

26. Close WH, Mount LE. Energy retention in the pig at several environmental temperatures and levels of feeding. Proc Nutr Soc. 1971;30(1):33A-34A.

27. Close $\mathrm{WH}$. The effects of plane of nutrition and environmental temperature on the energy metabolism of the growing pig. 3 . The efficiency of energy utilization for maintenance and growth. Br J Nutr. 1978;40(3):433-8.

28. Renaudeau D, Gourdine JL, St-Pierre NR. A meta-analysis of the effects of high ambient temperature on growth performance of growing-finishing pigs. J Anim Sci. 2011;89(7):2220-30.

29. Christon R. The effect of tropical ambient temperature on growth and metabolism in pigs. J Anim Sci. 1988;66(12):3112-23.

30. Zhang XD. Effect of heat stress on physiological and biochemical responses of pigs. M.S. Thesis. Shandong Agricultural University, Taian, China, 2004.

31. Qu H, Ajuwon KM. Metabolomics of heat stress response in pig adipose tissue reveals alteration of phospholipid and fatty acid composition during heat stress. J Anim Sci. 2018;96(8):3184-95.

\section{Figures}

(A)

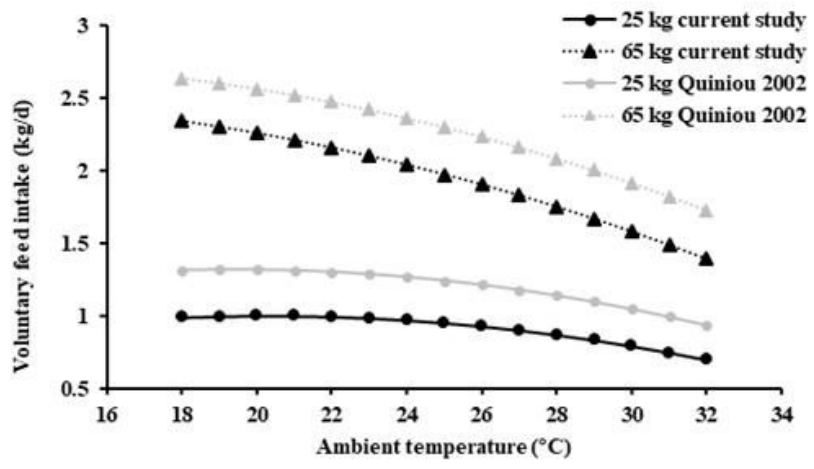

(C)

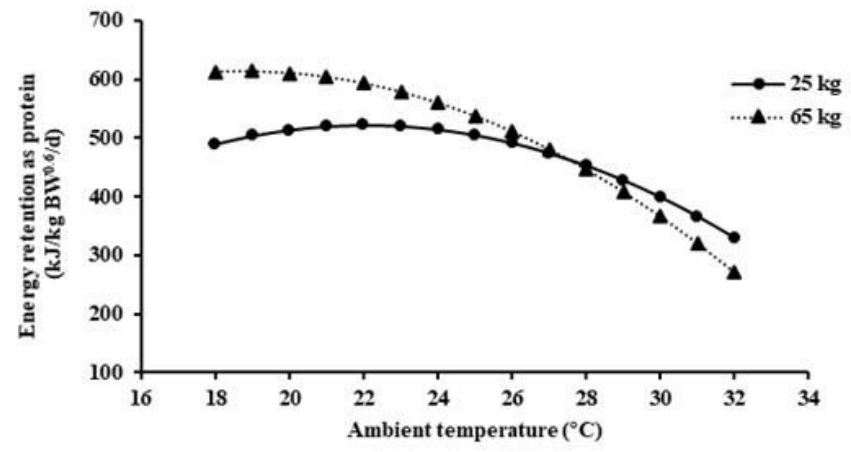

(B)

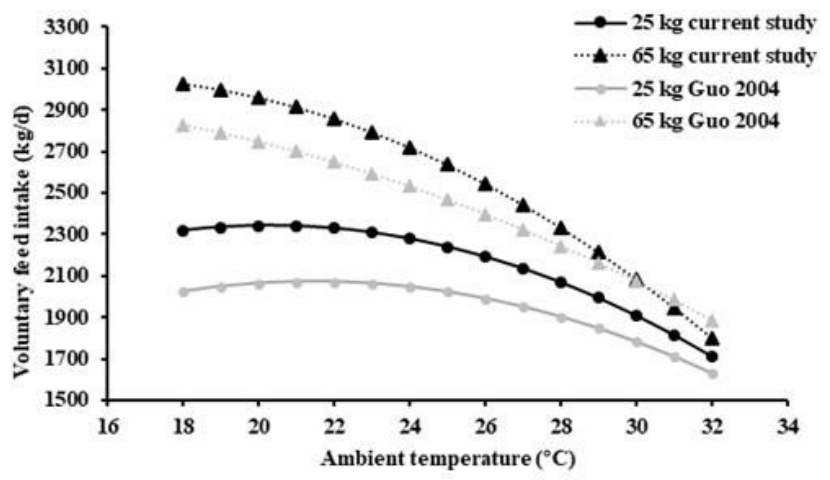

(D)

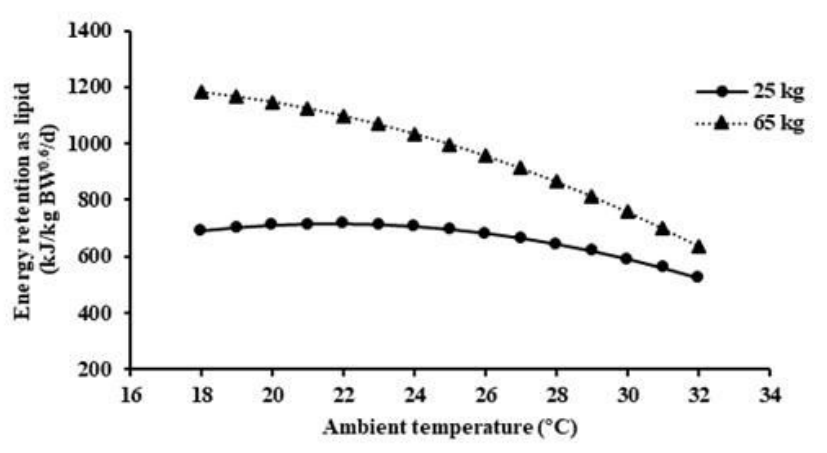

Figure 1

Modelling feed intake, energy intake and energy retention of pigs at $25 \mathrm{~kg}$ and $65 \mathrm{~kg}$ under ambient temperatures from $18^{\circ} \mathrm{C}$ to $32^{\circ} \mathrm{C}$. (A) Modelling the voluntary feed intake (dark curves) and comparison with models developed previously by Quiniou et al. [20] (grey curves). 
(B) Modelling the metabolizable energy intake (dark curves) and comparison with models developed previously by Guo et al. [21] (grey curves). (C) Modelling the energy retention as protein. (D) Modelling the energy retention as lipid.
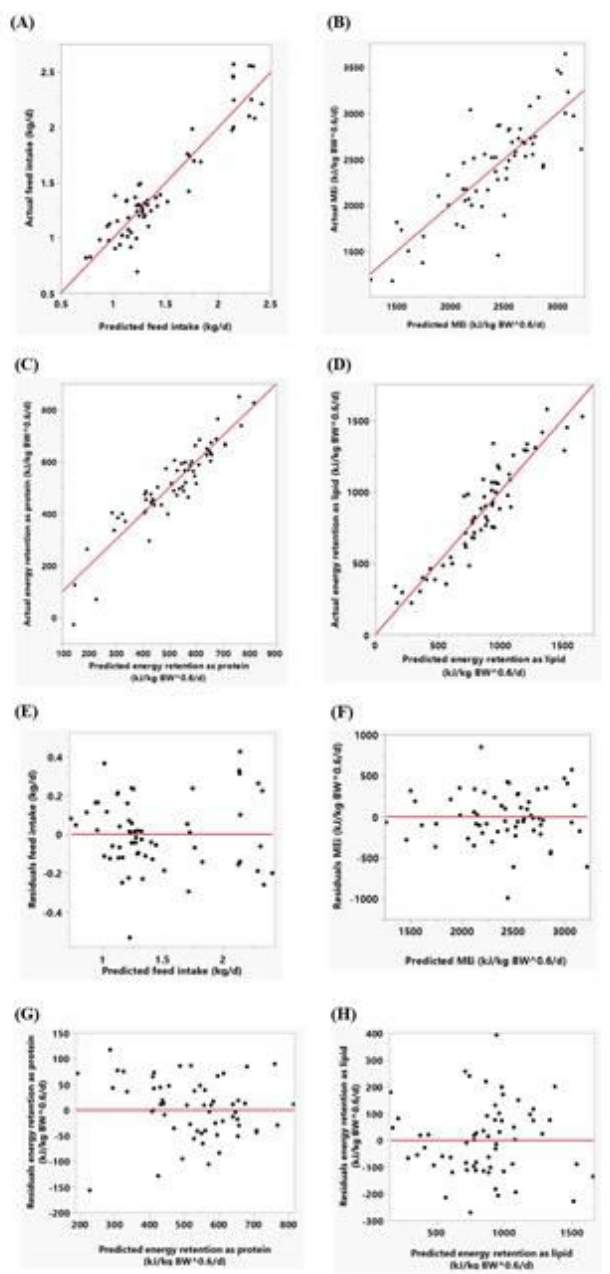

\section{Figure 2}

Test the goodness-of-fit of models developed in the current study. (A) Plot of predicted voluntary feed intake vs. actual voluntary feed intake. (B) Plot of predicted metabolizable energy intake vs. actual metabolizable energy intake. (C) Plot of predicted energy retention as protein vs. actual energy retention as protein. (D) Plot of predicted energy retention as lipid vs. actual energy retention as lipid. (E) Plot of predicted voluntary feed intake vs. voluntary feed intake residuals. (F) Plot of predicted metabolizable energy intake vs. metabolizable energy intake residuals. (G) Plot of predicted energy retention as protein vs. energy retention as protein residuals. (H) Plot of predicted energy retention as lipid vs. energy retention as lipid residuals. 


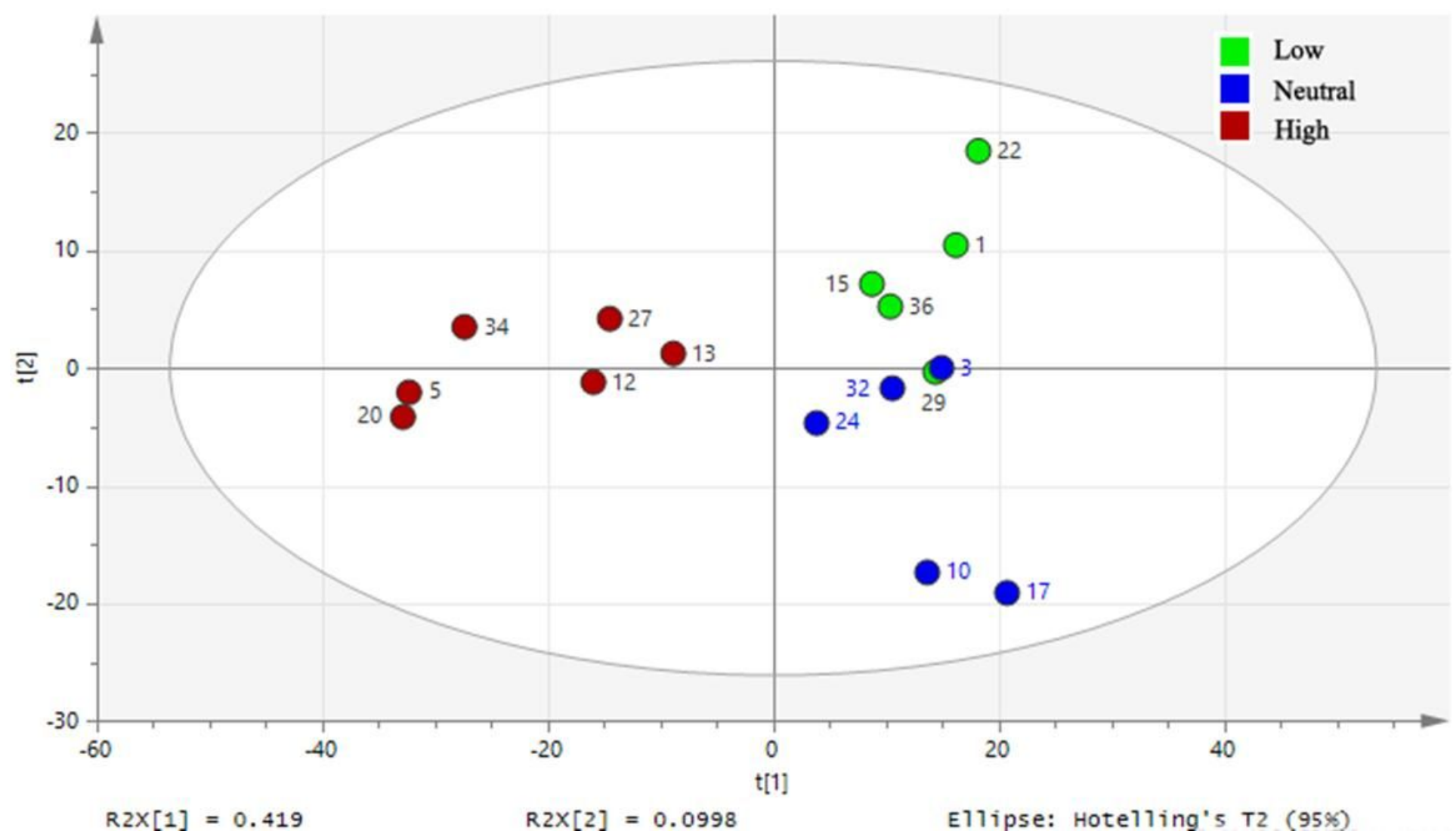

Figure 3

Principle component analysis (PCA) score plot demonstrating the separation of the plasma samples from pigs at $25 \mathrm{~kg}$ kept under different ambient temperatures. Low: $18^{\circ} \mathrm{C}$; Neutral: $23^{\circ} \mathrm{C}$; High: $32^{\circ} \mathrm{C}$. Each circle represents an individual plasma sample. 

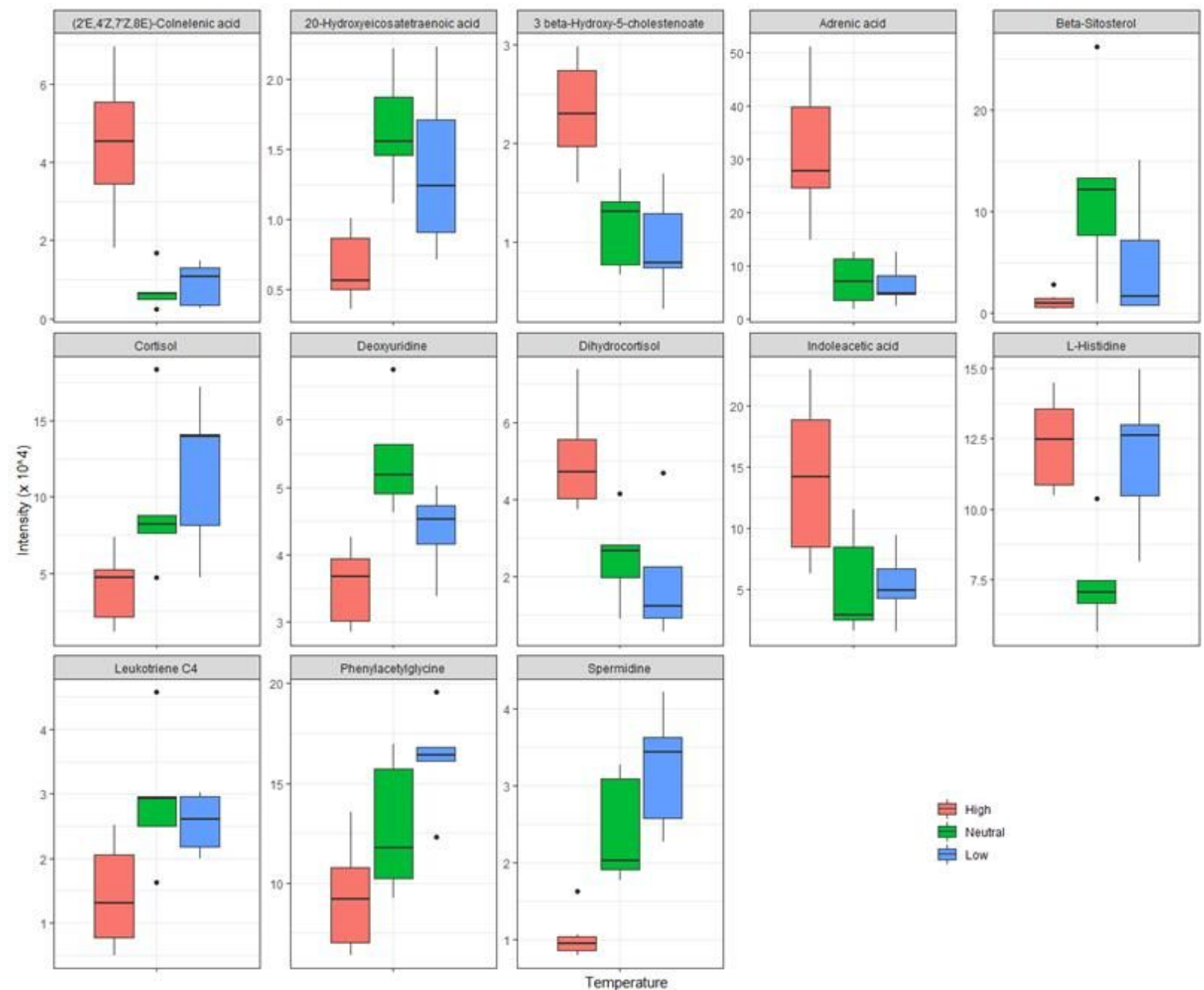

$$
\begin{aligned}
& \text { 岸 High } \\
& \text { Neutral } \\
& \text { 帛 Low }
\end{aligned}
$$

\section{Figure 4}

Identified compounds through metabolomics analysis with different intensities in plasma samples from pigs at $25 \mathrm{~kg}$ kept under different ambient temperatures. Low: $18^{\circ} \mathrm{C}$; Neutral: $23^{\circ} \mathrm{C}$; High: $32^{\circ} \mathrm{C}$. 